\title{
Attenuation of Oscillatory Pressures in Instrument Lines
}

\author{
By Arthur S. Iberall
}

\begin{abstract}
A theoretical investigation has been made of the attenuation and lag of an oscillatory pressure variation applied to one end of a tube, when the other end is connected to a pressuresensitive element.

An elementary theory based on incompressible viscous-fluid flow is first developed. The elementary solution is then modified to take into account compressibility; finite pressure amplitudes; appreciable fluid acceleration; and finite length of tubing (end effects). Account is taken of heat transfer into the tube.

The complete theory is derived in an appendix. The results are summarized in eight graphs in a form convenient for use in computing the lag and attenuation of a sinusoidal oscillation in a transmission tube.
\end{abstract}

\section{Introduction}

In many industrial processes, it is necessary to know or to utilize the pressure at one or more points in a fluid conduit. It is not always possible to connect an instrument directly into the conduit at those points. Instead, recourse must be had to remote indication or control. In the case that a fluid is used for transmitting the pressure, it is often of interest to the designer or user of such systems to know their response to variations in pressure. At the present time, the only solution easily available to the engineer is generally based on an elementary theory that considers the system as equivalent to an $\mathrm{R}-\mathrm{C}$ electrical network. (See, for example, NACA Technical Note 593, Pressure drop in tubing in aircraft instrument installations, by W. A. Wildhack.) The main defect of the theory is that it does not provide criteria for the limits of its applicability.

In the present paper, a relatively complete treatment is given for the transmission of oscillatory pressures in tubing. Primary consideration is given to simplifying the design of high-quality transmission systems for relatively low frequencies.

The elementary solution is derived and then extended to apply for oscillatory pressures that are an appreciable fraction of the absolute mean pressure, for appreciable frequencies of oscillation, and for tubing short enough to require end cor-

1 This work was supported by the Office of Naval Research under a project on "Basic Instrumentation for Scientific Research."

Attenuation of Pressure in Tubes rections. The effect of heat transfer in modifying the oscillatory response of the tube is also discussed.

The chief utility of knowing these corrections is that it permits the designer to choose the size of tubing for specific applications with greater confidence than can otherwise be done.

In the next section, the elementary theory of transmission lags is developed, and the corrections are discussed. The complete theory is presented in graphical form for the convenience of the designer. A number of examples of the use of the design charts are also given. This section is then followed by a mathematical appendix in which the more exact results are derived. All mathematical symbols used in this paper are defined in section II and also when they are first used.

\section{List of Mathematical Symbols}

$A=$ tube area.

$C=$ velocity of sound.

$D=$ inside diameter of tube.

$E=$ elastic modulus of tube.

$F=$ correction functions.

$K=$ thermal conductivity of fluid.

$L=$ tube length.

$M=$ mass flow.

$N=$ dimensionless parameter of fluid regime.

$Q=$ volumetric flow. 
$R=$ a volume ratio.

$R_{e}=$ Reynolds number.

$T=$ absolute temperature.

$V=$ instrument volume.

$b=$ compressibility factor for liquid.

$c=$ any arbitrary constant.

$c_{p}=$ specific heat of fluid.

$f=$ any arbitrary function.

$g=\mathrm{a}$ Bessel function argument.

$h=\mathrm{a}$ Bessel function argument.

$k=$ compressibility of a liquid.

$l=$ entrance length.

$m=$ exponent of "polytropic" expansion in instrument volume.

$n=$ exponent of "polytropic" expansion in tube.

$p=$ pressure.

$s=$ tube wall thickness.

$t=$ time.

$u=$ axial velocity.

$x=$ axial distance along tube.

$y=$ a dimensionless axial distance variable.

$z=$ dimensionless parameter of fluid regime.

$\gamma=$ ratio of specific heats.

$\delta=$ phase angle.

$\eta=$ density ratio.

$\lambda=$ time constant.

$\mu=$ fluid viscosity.

$\nu=$ kinematic viscosity.

$\xi=$ fractional pressure excess.

$\rho=$ fluid density.

$\sigma=$ Prandtl number.

$\phi=$ velocity potential.

$\chi=$ attenuation factor.

$\psi=$ attenuation parameter.

$\omega=$ angular frequency.

\section{Elementary Theory}

Figure 1 is a schematic drawing of the system that will be discussed throughout the paper. A tube transmits fluid pressure from a conduit to a pressure-sensitive instrument. The conduit applies an oscillatory (sinusoidal) pressure to the entrance of the transmission tube. The tube, which transmits the pressure, is characterized by a constant cross-sectional area and its length. The pressure-sensitive instrument, which receives the pressure, is characterized by its enclosed volume. It is assumed that if the walls enclosing the instrument volume are flexible (either elastic or piston-like), the enclosed volume can be re- placed by a larger equivalent rigid volume that will store the same mass of fluid per unit pressure change. It is further assumed that the pressuresensitive instrument will be so chosen that its indication is independent of the frequency of expected pressure oscillations.

In deriving the elementary theory, it is assumed that Poiseuille's law of viscous resistance holds at each point in the tube; that the fluid is incompressible in the tube; that the sinusoidal pressure oscillations at the beginning of the tube are of small amplitude compared to the mean absolute pressure; and that, if the fluid is a gas, it expands and contracts isothermally in the instrument volume.

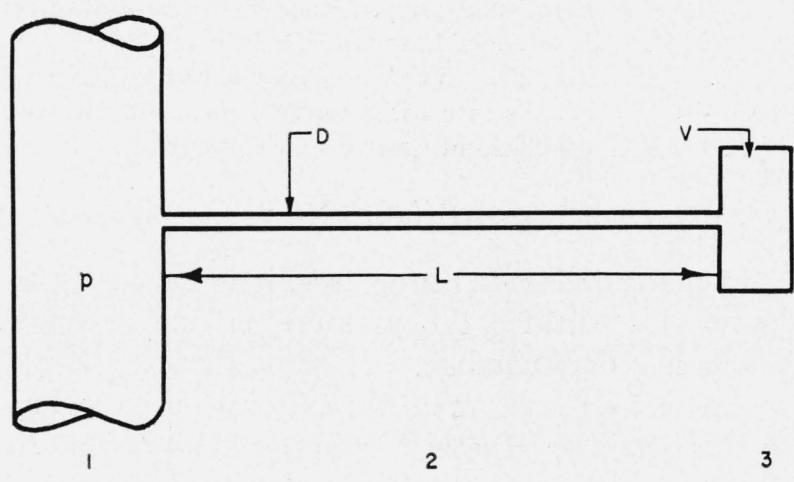

FIgure 1. Schematic diagram of a fluid transmission system (1-conduit, 2-transmission tube, 3-pressure instrument).

$$
p=p_{0}+\Delta p \cos \omega t .
$$

The same assumptions applied to an incompressible fluid (e.g., a liquid) lead to the conclusion that there is no loss in amplitude or lag in a liquidfilled system as a liquid would not expand or contract in the instrument volume.

We may write

$$
\frac{\partial p}{\partial x}=-\frac{128}{\pi} \frac{\mu_{0}}{D^{4}} Q
$$

for Poiseuille's law, and

$$
\frac{\partial M}{\partial x}=-A \frac{\partial \rho}{\partial t}
$$

for the equation of continuity. Here

$p=$ instantaneous pressure at any point in the tube

$x=$ distance along the tube measured from its entrance 
$\mu_{0}=$ mean fluid viscosity

$D=$ tube diameter

$Q=$ volumetric flow at any point in the tube

$\rho=$ instantaneous density at any point in the tube

$M=$ mass flow at any point in the tube

$A=$ cross-sectional area of the tube

$t=$ time.

We infer from the equation of continuity and the assumption that the fluid is incompressible in the tube (i. e., $\partial \rho / \partial t=0$ ) that the mass flow, and therefore the volumetric flow, does not vary along the tube, but at most varies only with time (the fluid motion is piston-like).

By differentiating eq 1, we then obtain

$$
\frac{\partial^{2} p}{\partial x^{2}}=0
$$

along the tube.

Our boundary conditions are that at $x=0$

$$
p=p_{0}+\Delta p e^{j \omega t},
$$

a sinusoidal pressure variation about the mean pressure, and that at $x=L$

$$
\left.\begin{array}{c}
Q=\frac{V}{p_{0}} \frac{\partial p}{\partial t} \\
\frac{\partial p}{\partial x}=-\frac{128}{\pi} \frac{\mu_{0}}{D^{4}} Q
\end{array}\right\} .
$$

The first line of eq 5 expresses the rate at which a compressible fluid entering a rigid volume builds up pressure, whereas the second line of eq 5 states that the flow into the volume is limited by the pressure gradient at the end of the tube. Here

$p_{0}=$ mean pressure at the entrance

$\Delta p=$ amplitude of the pressure oscillation at the conduit

$V=$ instrument volume

$\omega=$ angular frequency of the pressure oscillation

$L=$ length of the tube.

It is convenient to introduce a new variable $\xi$, the fractional pressure excess, defined as

$$
\xi=\frac{p-p_{0}}{p_{0}}
$$

so that eq 3,4 , and 5 become, respectively,

$$
\frac{\partial^{2} \xi}{\partial x^{2}}=0
$$

at $x=0$

$$
\xi=\xi_{0} e^{j \omega t}
$$

and at $x=L$,

$$
\left.\begin{array}{rl}
\frac{\partial \xi}{\partial x} & =-\frac{128}{\pi} \frac{\mu_{0}}{p_{0}} \frac{V}{D^{4}} \frac{\partial \xi}{\partial t} \\
& =-\frac{\lambda_{0}}{L} \frac{\partial \xi}{\partial t}
\end{array}\right\} .
$$

where

$$
\left.\begin{array}{rl}
\lambda_{0} & =\frac{128}{\pi} \frac{\mu_{0}}{p_{0}} \frac{L}{D^{4}} V \\
& =32 \frac{\mu_{0}}{p_{0}}\left(\frac{L}{D}\right)^{2} \frac{V}{A L}
\end{array}\right\} .
$$

Here

$\xi=$ fractional pressure excess

$\xi_{0}=$ amplitude of the fractional pressure excess at the origin $\left(=\Delta p / p_{0}\right)$

$\lambda_{0}=$ a time constant of the system.

It is of further convenience to separate the pressure excess into a part that varies with $x$ and one that varies with $t$.

Let

$$
\xi=\tilde{\xi} e^{j \omega t},
$$

where $\tilde{\xi}$ is the maximum amplitude of the pressure excess at any point of the tube.

Our equations then become

$$
\frac{d^{2} \tilde{\xi}}{d x^{2}}=0
$$

at $x=0$,

$$
\tilde{\xi}=\xi_{0},
$$

and at $x=L$

$$
\frac{d \tilde{\xi}}{d x}=-\frac{\lambda_{0} \omega}{L} j \tilde{\xi}
$$


The solution of eq 12, which satisfies eq 13 and 14 , is

$$
\tilde{\xi}=\xi_{0} \frac{1+\lambda_{0} \omega\left(1-\frac{x}{L}\right) j}{1+\lambda_{0} \omega j} .
$$

The ratio of the amplitude of the pressure excess at the end of the tube to that at the beginning of the tube is then given by

$$
\left.\begin{array}{rl}
\frac{\tilde{\xi}_{L}}{\xi_{0}} & =\frac{1}{1+\lambda_{0} \omega j} \\
& =\frac{1}{1+\chi_{0} j}
\end{array}\right\}
$$

where

Here

$$
\chi_{0}=\lambda_{0} \omega
$$

$\tilde{\xi}_{L}=$ maximum amplitude of the pressure excess at the instrument volume

$\chi_{0}=$ an attenuation factor.

The real part of eq 16 is the attenuation in amplitude of the pressure excess, whereas the imaginary part is the phase lag, or

$$
\left.\begin{array}{l}
\left|\frac{\tilde{\xi}_{L}}{\xi_{0}}\right|=\frac{1}{\left[1+\chi_{0}^{2}\right]^{\frac{1}{2}}} \\
\tan \delta_{0}=\chi_{0},
\end{array}\right\},
$$

where $\delta_{0}$ is the lagging phase angle.

We will regard eq 18 as the elementary solution of our problem. It indicates that a transmission system is characterized by a time constant $\lambda_{0}$, which can be computed from a knowledge of the dimensions of the tube, the internal volume of the end device, and the average conditions of the gas in the tube; and an attenuation factor $\chi_{0}$, for each angular frequency, from which one can compute the attenuation and phase lag in a tube. The tube dimensions and the instrument volume furnish the analog to the resistance and capacitance of an electrical network.

In principle, although difficult in practice, from a knowledge of the response to a sine wave, one can obtain the response to square waves, step function, etc., by Fourier analysis.

\section{Discussion of Corrections}

The assumptions made in the elementary theory are restrictive, and in the appendix we shall modify them, one at a time, until finally we arrive at a complete solution that accurately takes into account all first-order phenomena, and partially takes into account second-order phenomena. Complete results are presented in convenient graphical form in figures 2 to 9 .

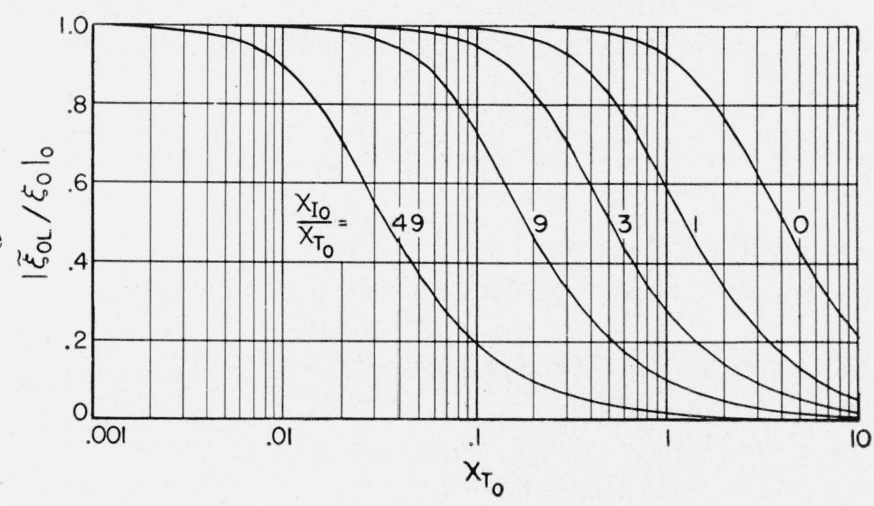

Figure 2. Amplitude ratio of the fundamental $\left|\tilde{\xi}_{O L} / \xi_{0}\right|_{0}$ in a volume terminated tube as a function of a parameter proportional to frequency $\left(\chi_{T_{0}}\right)$ for various ratios of instrument volume to tube volume $\left(\chi_{I_{0}} / \chi_{T_{0}}\right)$ with large damping $(z \leq 1)$.

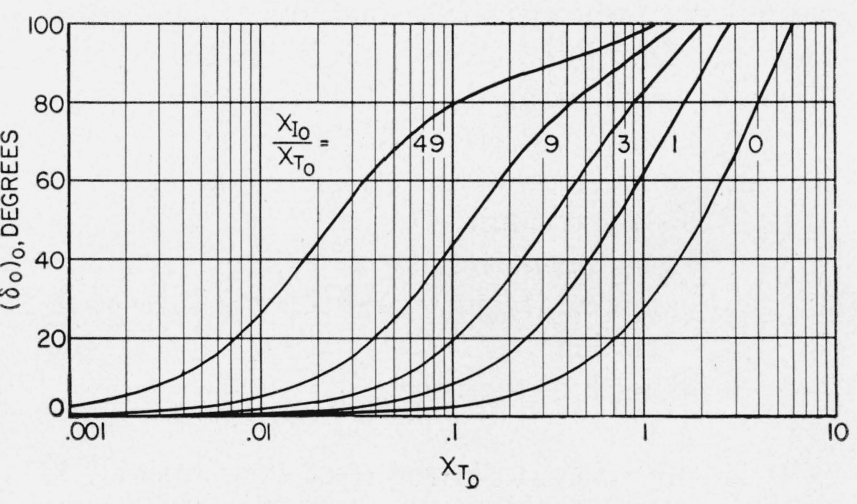

Figure 3. Phase lag of the fundamental $\left(\delta_{o}\right)_{0}$ in a volume. terminated tube as a function of a parameter proportional to frequency $\left(\chi_{T 0}\right)$ for various ratios of instrument volume to tube volume $\left(\chi_{T 0} / \chi_{T 0}\right)$ with large damping $(z \leq 1)$. 


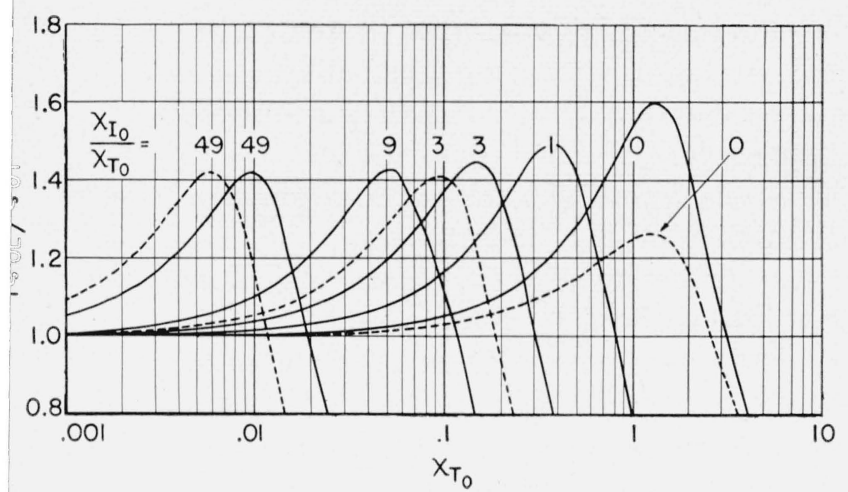

Figure 4. Amplitude ratio of the fundamental $\left|\tilde{\xi}_{O L} / \xi_{0}\right|$ in a volume terminated tube as a function of a parameter proportional to frequency $\left(\chi_{T 0}\right)$ for various ratios of instrument volume to tube volume $\left(\chi_{I_{0} / \chi_{T 0}}\right)$ and for two values of specific heat ratio $(\gamma)$ with intermediate damping $(z=$ $6.25)$.

$$
\longrightarrow, \gamma=1 ;-\cdots, \gamma=2 \text {. }
$$

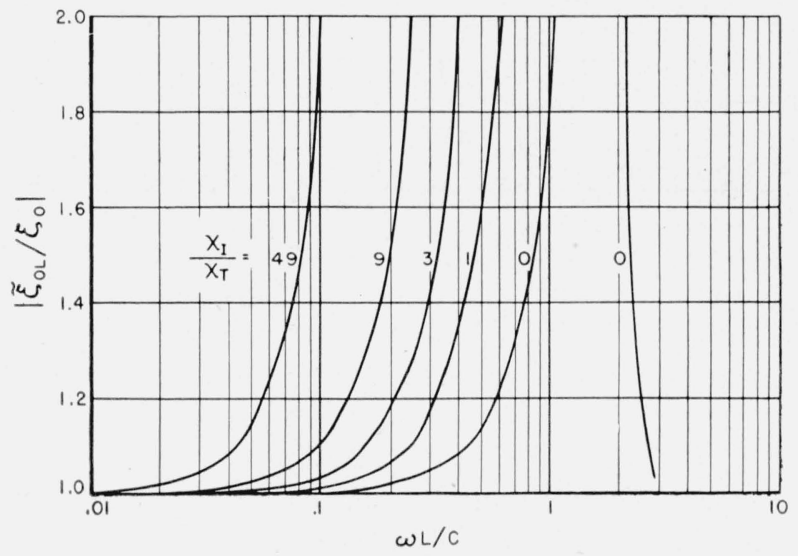

Figure 6. Amplitude ratio of the fundamental $\left|\tilde{\xi}_{O L} / \xi_{0}\right|$ in a volume terminated tube as a function of a parameter proportional to frequency $(\omega L / C)$ for various ratios of instrument volume to tube volume $\left(\chi_{I} / \chi_{T}\right)$ with little damping $(z \geq 100)$.

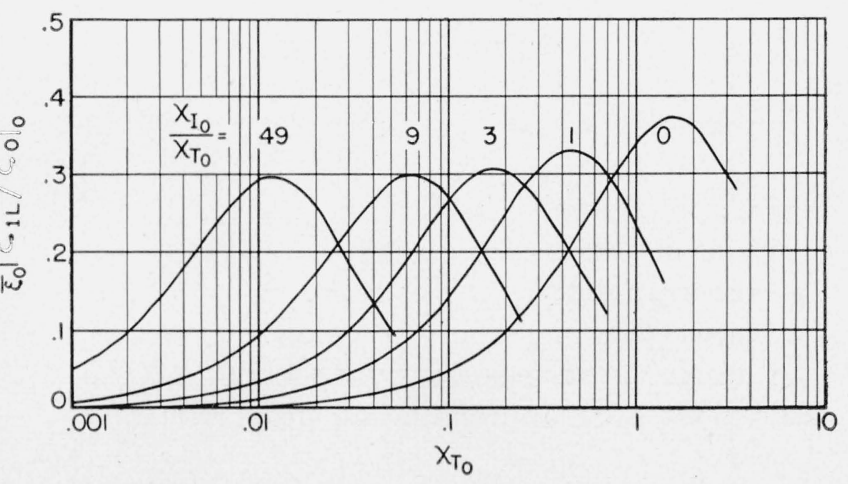

Attenuation of Pressure in Tubes

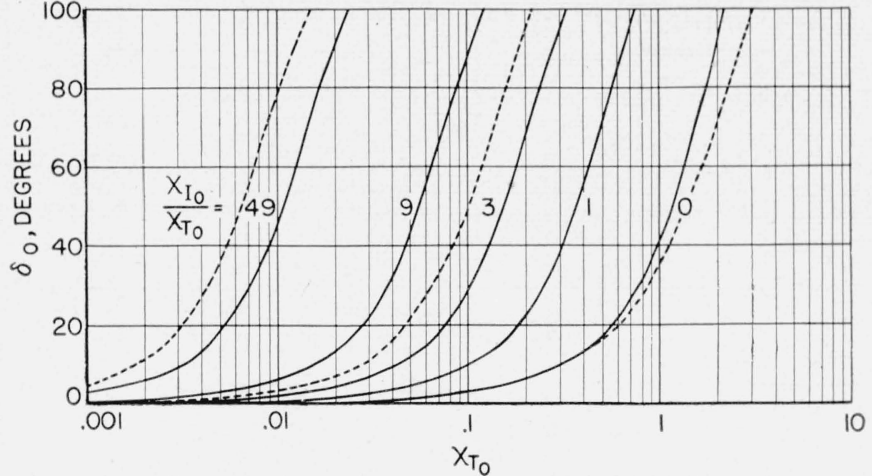

Figure 5. Phase lag of the fundamental ( $\left.\delta_{0}\right)$ in a volume terminated tube as a function of a parameter proportional to frequency $\left(\chi_{T 0}\right)$ for various ratios of instrument volume to tube volume $\left(\chi_{\mathrm{I} 0} / \chi_{T 0}\right)$ and for two values of specific heat ratio $(\gamma)$ with intermediate damping $(z=6.25)$.

$$
\longrightarrow, \gamma=1 ;--, \gamma=2 \text {. }
$$

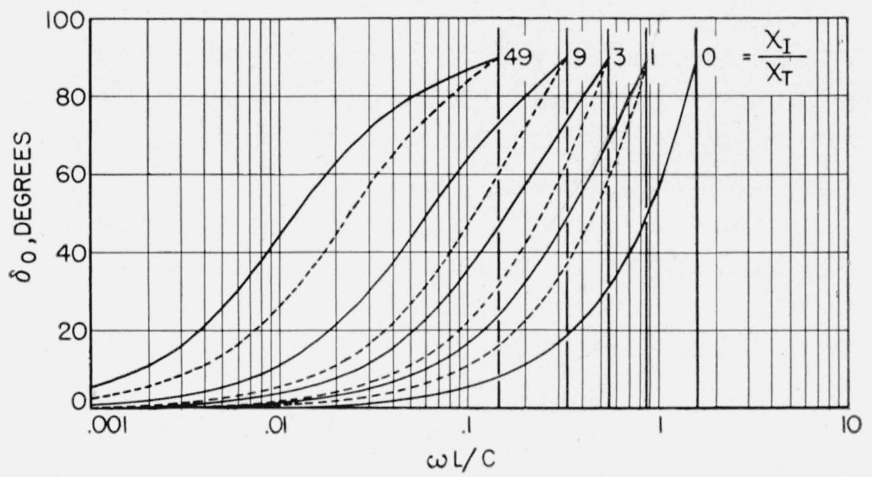

Figure 7. Phase lag of the fundamental ( $\left.\delta_{0}\right)$ in a volume terminated tube as a function of a parameter proportional to frequency $(\omega L / C)$ for various ratios of instrument volume to tube volume $\left(\chi_{I} / \chi_{T}\right)$ indicating the difference between no damping $\left(\left[\gamma \chi_{T 0} / 16\right]^{1 / 2}=0\right)$ and small damping $\left(\left[\gamma \chi_{T 0} / 16\right]^{1 / 2}=1\right)$ for two values of specific heat ratio $(\gamma)$ with small damping $(z \geq 100)$.

$\longrightarrow, \gamma=1 ;---\gamma=2$. For curved lines $\sqrt{\frac{\gamma \chi_{T O}}{16}}=1$; for solid straight lines $\sqrt{\frac{\gamma \chi_{T o}}{16}}=0$.

FIgURe 8. Relative amplitude of the double harmonic distortion $\left(\left|\xi_{1 L} / \xi_{0}\right|_{0} / \xi_{0}\right)$ in a volume terminated tube as a function of a parameter proportional to frequency $\left(\chi_{T 0}\right)$ for various ratios of instrument volume to tube volume $\left(\chi_{I 0} / \chi_{T 0}\right)$ with large damping $(z \leq 1)$. 


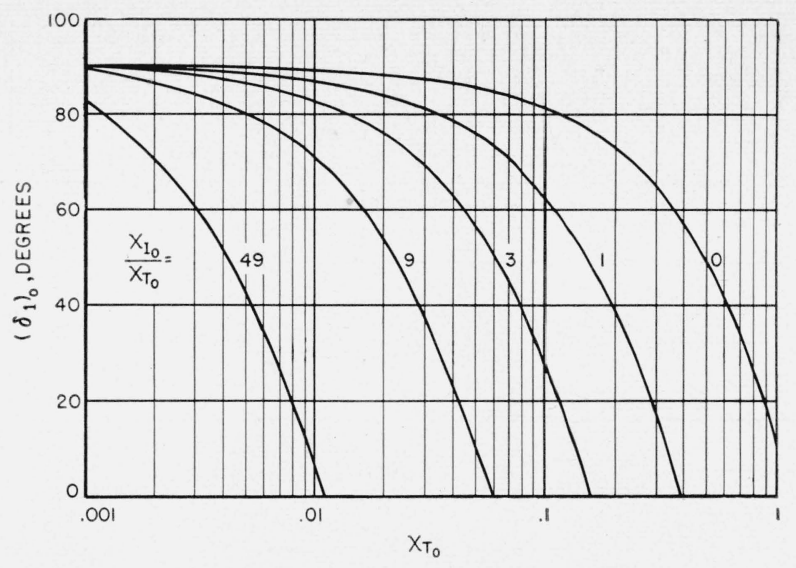

Figure 9. Phase lead (as measured on the fundamental time scale) of the double harmonic distortion $\left(\delta_{1}\right)_{0}$ in a volume terminated tube as a function of a parameter proportional to frequency $\left(\chi_{T 0}\right)$ for various ratios of instrument volume to tube volume $\left(\chi_{I_{0} / \chi_{T 0}}\right)$ with large damping $(z \leq 1)$.

The factors that must be taken into account are:

1. Compressible flow in the tube. The effect of fluid compressibility is to introduce a time constant and corresponding attenuation factor $\left(\lambda_{T}, \chi_{T}\right)$ depending on the tube volume in addition to the ones depending on the instrument volume. (The time constant and attenuation factor depending on the instrument volume will be referred to as $\lambda_{I}$ and $\chi_{I}$ henceforth instead of $\lambda_{0}$ and $\chi_{0}$ ). In terms of the electrical analog, the tube volume represents a distributed capacitance in addition to the equivalent capacitance of the instrument volume.

2. Finite pressure excess. The effect of the application of a finite pressure excess to a compressible fluid in a transmission tube is to introduce harmonic distortion and to modify the mean pressure. However, the attenuation of the fundamental is essentially independent of the magnitude of the pressure excess. The percentage of distortion is approximately proportional to the applied pressure excess.

3. Fluid acceleration. The effect of fluid inertia is to modify the time constants of the system. Both the attenuation of the fundamental and the magnitude of harmonic distortion are affected. A dimensionless parameter $z$ analagous to the " $Q$ " of an electrical system characterizes the fluid regime and determines whether fluid inertia may or may not be neglected.

When fluid inertia is negligible, a transmission tube acts like a highly damped system; when fluid inertia is large a transmission tube acts like an undamped system, and elementary acoustic theory is applicable.

4. Finite length of tubing. The effect of fluid acceleration at the ends of the tube results in further distortion of wave form, which must be taken into account in short tubes.

5. Heat conduction. If there were no heat transfer from outside the tube to inside, the oscillatory processes would take place adiabatically; if there were perfect heat transfer into and through the tube, the processes would take place isothermally. The effect of finite heat conduction is to make the real process occur in between these extremes, although in a rather complicated fashion. At low frequencies the process may be regarded as isothermal.

Although an exact result is given in the appendix, it is advantageous to utilize the thermodynamic equation of condition, discussed in the following section, for elucidating the problem of attenuation in tubing.

\section{Thermodynamic Equation of Condition}

In the case of an oscillatory variation of fluid flow, the equation relating the thermodynamic parameters of the fluid lie between the adiabatic and the isothermal equations of condition. For high frequencies, as in sound waves, it is well known that the adiabatic equation holds. However, for viscously damped motion, the adiabatic relation is not, in general, attained.

For a gas, we assume and justify in the appendix the processes can be described as "polytropic", that is, characterized by a constant exponent $n$, in the expression

with

$$
\left.\begin{array}{rl}
p & =c \rho^{n} \\
1+\xi & =\eta^{n}
\end{array}\right\}
$$

where

$$
1 \leqq|n| \leqq \gamma,
$$

$n=$ exponent of the "polytropic" expansion in the tube

$\gamma=$ ratio of specific heats

$\eta=$ density ratio $\left(\rho / \rho_{0}\right)$

$\rho_{0}=$ average density in the tube.

$c$ is used to indicate any constant.

The viscosity of gases is independent of the pressure, and, as an approximation, proportional to 
the absolute temperature. (The more rigorous approximation is that the viscosity is proportional to $[T]^{1 / 2} /[1+c / T]$ but over a small range this can be approximated by the temperature to a power close to one. For example, for air at room temperature, a power of 0.8 fits experimental data quite well. The difference from unity is unimportant for our purpose.)

Therefore,

$$
\left.\begin{array}{rl}
\mu & =c T, \\
\frac{\mu}{\mu_{0}} & =(1+\xi)^{\frac{n-1}{n}}, \\
\frac{\mu}{\mu_{0}} & =\eta^{n-1},
\end{array}\right\}
$$

follows from the gas laws and eq 19. Here $\mu$ is the instantaneous fluid viscosity. and $T$ is the absolute temperature.

Equations 19 and 20 thus express the variation of viscosity, density, and pressure in a polytropic process in a gas. At low frequencies, the polytropic exponent may be taken as equal to unity.

For liquids, we assume that the equation of condition in a polytropic process is given by

where

$$
\rho=\rho_{0}+c p^{n},
$$

$$
1 \leqq|n| \leqq \gamma .
$$

For liquids, however, $\gamma$ lies so close to unity that we may satisfactorily assume $n=1$.

Equation 21 can then be written in the form

$$
\eta=1+b \xi,
$$

where $b=$ a compressibility factor $\left(=k_{0} p_{0}\right)$

$k_{0}=$ liquid compressibility at average conditions in the tube.

The variation in viscosity of a liquid over a small range of temperature can be neglected, so that in a polytropic process

$$
\mu=\mu_{0} .
$$

Actually the implication in eq 22 and 23 is that in a liquid-filled transmission line, the effect of conditions appreciably different from isothermal is negligible.

It is also necessary to take into account heat exchange at the pressure element.

For an isothermal process with a gas in the instrument volume, we previously assumed that

$$
Q=\frac{V}{p_{0}} \frac{\partial p}{\partial t}
$$

represents the influx of fluid. If, instead, a polytropic process in the instrument is assumed, characterized by an exponent, $m$ (the heat exchange may differ in the tube and instrument volume so that $m$ is not necessarily equal to $n$ ), then eq. 5 should be modified to

$$
\left.\begin{array}{l}
Q=\frac{V}{m p} \frac{\partial p}{\partial t} \\
Q=\frac{n}{m} \frac{V}{\rho} \frac{\partial \rho}{\partial t}
\end{array}\right\}
$$

in the case of gases; or to

for liquids.

$$
Q=\frac{V b}{p_{0}} \frac{\partial p}{\partial t}
$$

If the fluid is regarded as a spring, the exponent of the polytropic process for a gas, or the compressibility of a liquid may be viewed as quantities that make the fluid spring stiffer in the case of gases, or almost infinitely stiff in the case of liquids. It -is shown in the appendix that these polytropic exponents modify the time constants of the tube and volume.

\section{General Procedure, with Examples, for Computing Transmitted Pressure}

The computation of the attenuation and phase lag at one end of a transmission tube of a sinusoidal pressure variation imposed at the other end can be carried out with the aid of figures 2 to 9, These figures are based upon the theory largely developed in the appendix. The computations are made primarily for the attenuation at the fundamental frequency. An estimate of the distortion arising from finite input amplitudes with high damping is made in the appendix. The computation for the first harmonic in the distorted output can be made with the aid of figures 8 and 9 . An outline of procedure for making computations follows.

1. Compute

$$
z=\frac{D^{2}}{4} \frac{\omega}{\nu_{0}}
$$

a dimensionless parameter of the fluid regime that characterizes the amount of damping present. When this parameter is less than 1 (large damping), use figures 2 and 3 ; when greater than 100 (small damping), use figures 6 and 7 . For inter- 
mediate values of this parameter, use figures 4 and 5 as an aid to interpolation.

2. Compute the attenuation factors

$$
\begin{gathered}
\chi_{T_{0}}=\frac{32 \mu_{0} \omega}{p_{0}}\left(\frac{L}{D}\right)^{2}, \\
\frac{\chi_{I_{0}}}{\chi_{T_{0}}}=\frac{1}{m}\left(\frac{V}{A L}\right),
\end{gathered}
$$

for a gas, or

$$
\begin{gathered}
\chi_{T_{0}}=32 \mu_{0} k_{0} \omega\left(\frac{L}{D}\right)^{2}\left(1+\frac{p_{0}-p_{a}}{p_{0}} \frac{1}{k_{0} E} \frac{D}{s}\right), \\
\frac{\chi_{I_{0}}}{\chi_{T_{0}}}=\frac{V}{A L} \frac{1}{\left(1+\frac{p_{0}-p_{a}}{p_{0}} \frac{1}{k_{0} E} \frac{D}{s}\right)},
\end{gathered}
$$

for a liquid. These quantities, $\chi_{T 0}$ and $\chi_{I 0}$, are factors based on the tube volume and instrument volume, respectively. The zero subscript means that they are values for the case of large damping.

3. Compute the input pressure excess

$$
\xi_{0}=\frac{\Delta p}{p_{0}} .
$$

4a. For values of $z$ less than 1, enter figure 2 with $\chi_{T 0}$ and $\chi_{T_{0}} / \chi_{T 0}$ to find the amplitude ratio $\left|\tilde{\xi}_{o L} / \xi_{0}\right|_{0}$ and enter figure 3 to find the lagging phase angle $\left(\delta_{O}\right)_{0}$.

$4 \mathrm{~b}$. The output pressure excess is then computed from

$$
\left|\tilde{\xi}_{O L}\right|_{0}=\xi_{0}\left|\frac{\tilde{\xi}_{O L}}{\xi_{0}}\right|_{0}
$$

5a. For values of $z$ greater than 100, compute

$$
\begin{aligned}
& \frac{\omega L}{C}=\left[\frac{z \chi_{T 0}}{8 \gamma}\right]^{\frac{1}{2}}, \\
& \frac{\chi_{I}}{\chi_{T}}=\gamma\left(\frac{\chi_{T 0}}{\chi_{T 0}}\right) .
\end{aligned}
$$

For liquids, assume $\gamma=1$.

The quantities $\chi_{I}$ and $\chi_{T}$ are the attenuation factors for the case of low damping. With low damping, it is convenient to use the dimensionless parameter $\omega L / C$, which is proportional to frequency, as the independent variable.

$5 \mathrm{~b}$. Enter figure 6 with $\omega L / C$ and $\chi_{I} / \chi_{T}$ to find the amplitude ratio $\left|\tilde{\xi}_{O L} / \xi_{0}\right|$ and enter figure 7 to find the lagging phase angle $\delta_{o}$. It is necessary to estimate the phase angle by interpolation. For very small values of $\chi_{T 0}$ compared to 1 , the lagging phase angle is zero up to the first resonance. In figure 7 , curves have been presented to indicate the phase angle for $\left[\gamma \chi_{T 0} / 16\right]^{1 / 2}=0$ and $\left[\gamma \chi_{T 0} / 16\right]^{1 / 2}=1$ One may linearly interpolate between these curves on the basis of $\left[\gamma \chi_{T 0} / 16\right]^{1 / 2}$ for values lying between 0 and 1.

6a. For values of $z$ lying between 1 and 100 , one may interpolate between the values of amplitude ratio and lagging phase angle obtained in step $4 \mathrm{a}$ and those obtained in step $5 \mathrm{~b}$ by the use of figures 4 and 5 . Enter figures 4 and 5 with $\chi_{T 0}$ and $\chi_{T 0} / \chi_{T 0}$ to find the amplitude ratio $\left|\tilde{\xi}_{O L} / \xi_{0}\right|$ and lagging phase angle $\delta_{o}$. These are the values for $z=6.25$. In order to interpolate, plot a logarithmic graph with $z$ as abscissa and the amplitude ratio or phase angle as ordinate. Plot the values from step $4 \mathrm{a}$ at $z=1$, from step $6 \mathrm{a}$ at $z=6.25$, and from step $5 \mathrm{~b}$ at $z=100$, draw a curve through these three points, and interpolate on this curve for the intermediate value of $z$.

\section{Computation of Double Frequency Distortion}

1. This computation represents only an estimate of the double frequency distortion and is strictly valid only for values of $z$ less than 1 .

Compute $\chi_{T 0}$ and $\chi_{I 0} / \chi_{T 0}$. Enter figures 8 and 9 to obtain the relative amplitude ratio $\left|\tilde{\xi}_{1 L} / \xi_{0}\right|_{0} / \xi_{0}$ and leading phase angle $\left(\delta_{1}\right)_{0}$ for the double frequency wave. The leading phase angle is measured on the time scale of the fundamental, where both the fundamental and double frequency waves are cosine terms.

2. Compute the pressure excess of the double frequency $\left|\tilde{\xi}_{1 L}\right|_{0}$ from

$$
\left|\tilde{\xi}_{1 L}\right|_{0}=\left(\frac{1}{\xi_{0}}\left|\frac{\tilde{\xi}_{1 L}}{\xi_{0}}\right|\right)_{0} \xi_{0}^{2}
$$

The various quantities in the above section are defined below:

$\mu_{0}=$ mean fluid viscosity.

$\nu_{0}=$ mean kinematic viscosity.

$p_{0}=$ mean fluid pressure.

$p_{a}=$ ambient pressure external to the tube.

$\Delta p=$ amplitude of the applied sinusoidal pressure.

$\xi_{0}=$ applied fractional pressure excess.

$\tilde{\xi}_{\text {OL }}=$ pressure excess of the fundamental at the instrument volume.

$\tilde{\xi}_{1 L}=$ pressure excess of the double frequency at the instrument volume.

$\delta_{O}=$ lagging phase angle of the fundamental at the instrument volume.

$\delta_{1}=$ leading phase angle of the double frequency at the instrument volume. 
$k_{0}=$ mean fluid compressibility.

$V=$ equivalent rigid internal volume of the instrument.

$A=$ internal cross section of the tube.

$D=$ internal diameter of the tube.

$s=$ wall thickness of the tube (assumed small compared to the diameter).

$E=$ elastic modulus of the tube material.

$L=$ length of the tube.

$C=$ velocity of sound in the fluid.

$\gamma=$ ratio of specific heats of the fluid (assumed to be one for liquids).

$m=$ coefficient of the polytropic process in the instrument volume. (In lieu of other information, it may be assumed to be one.)

$z=$ dimensionless parameter characterizing the fluid regime.

$\omega=$ angular frequency applied.

$\chi_{T}=$ attenuation factor based on the tube volume.

$\chi_{I}=$ attenuation factor based on the instrument volume.

Subscript $T$ refers to parameters based on tube volume; subscript $I$ refers to parameters based on end volume; $O$ or 1 following a $T$ or $I$ denotes the fundamental or first harmonic; an end subscript of 0 denotes a value for the case of large damping.

The attenuation of the fundamental may be validly computed from the formulas developed in this paper when

$$
\left.\begin{array}{l}
\frac{\nu_{0}}{C D}<1 \\
\frac{\nu_{0} \omega}{C^{2}}<1
\end{array}\right\}
$$

The second harmonic distortion, which was only estimated approximately, may be validly computed from the formulas developed when

$$
z=\frac{\omega D^{2}}{4 \nu_{0}}<1,
$$

and when the applied pressure amplitude is sufficiently small at the applied frequency to permit laminar flow. 3. Compute the mean pressure in the instrument volume, which is larger than the mean pressure at the tube entrance by

$$
\xi_{0} \Delta p\left[1-\left|\tilde{\xi}_{O L} / \xi_{0}\right|_{0}^{2}\right] / 4
$$

\section{Examples of Computations}

The calculation of attenuation by the general procedure outlined above will be illustrated by a number of examples.

(a) What is the longest length of $3 / 16$-in.-insidediameter tubing that can be used to transmit air pressure to a Bourdon pressure gage (equivalent internal volume assumed negligible) up to a frequency of $1 / 2 \mathrm{c} / \mathrm{s}$ with a loss in amplitude not greater than 25 percent? What will be the double frequency distortion? For air assume $\mu_{0}=2 \times 10^{-4}$ poise, $\nu_{0}=1 / 6$ stokes, $m=1, \quad \gamma=1.4, \quad p_{0}=10^{6}$ dynes $/ \mathrm{cm}^{2}$ (atmospheric pressure), angular frequency $\omega=\pi$.

Using eq 26, $z=1.1$ (computed in consistent units). This value is sufficiently close to unity to permit the use of figures 2 and 3 . Enter figure 2 with $\left|\tilde{\xi}_{o L} / \xi_{0}\right|_{0}=0.75$ and $\chi_{T 0} / \chi_{T 0}=0$, since the instrument volume is negligible, to find $\chi_{T 0}=2.1$. Compute $L$ in eq 27 to be 160 feet.

Entering figure 3 with $\chi_{T 0}=2.1$, to find that the maximum phase lag will be 53 degrees.

Entering figure 8 to find that the relative amplitude of the double frequency $\left|\tilde{\xi}_{1 L} / \xi_{0}\right|_{0} / \xi_{0}=0.35$. For initial pressure excesses of $0.1,0.3$, and 1 , respectively, the double frequency amplitude, relative to the input amplitude, will be $3 \frac{1}{2}, 10 \frac{1}{2}$, and 35 percent, while the mean pressure will increase $0.0010,0.010$, and 0.11 of an atmosphere, respectively.

(b) What lengths of 0.1-in.-inside-diameter tubing (nominally $3 / 16$-in.-outside-diameter tubing) can be used for quality transmission of air pressure for frequencies up to $1,10,100,1,000 \mathrm{c} / \mathrm{s}$ into pressure instruments with equivalent rigid volumes of 0.1 and 1 in. ${ }^{3}$ ?

We will define quality transmission as that in which there is no more than \pm 5 -percent change in fundamental amplitude or more than $\pm 30^{\circ}$ phase shift (whichever is more stringent).

Assume that $\mu_{0}=2 \times 10^{-4}$ poise, $\nu_{0}=1 / 6$ stokes, $m=1, \gamma=1.4, D=0.1$ in., $A=0.0079$ in. $^{2}, p_{0}=10^{6}$ dynes $/ \mathrm{cm}^{2}, \rho_{0}=0.0012 \mathrm{~g} / \mathrm{cm}^{3}$.

We will calculate for each frequency separately. b (1). $f=1 \mathrm{c} / \mathrm{s}$ :

Using eq $26, z=0.61$; therefore, use figures 2 and 3 .

Assume $A L=\infty$, therefore, by eq $28, \chi_{10} / \chi_{T 0}=0$.

Enter figure 2 for $\left|\tilde{\xi}_{o L} / \xi_{0}\right|_{0}=0.95$ to find $\chi_{T 0}=$ 0.80 . 
Enter figure 3 for $\left(\delta_{O}\right)_{0}=30^{\circ}$ to find $\chi_{T 0}=1.1$; use 0.80 since it is more stringent.

Calculate $L$ from eq 27 to be 450 in.

Calculate $A L$ to be 3.5 in. $^{3}$

Compute $\chi_{I 0} / \chi_{T 0}$ from eq 28 to be 0.029 for $V=0.1$ in. ${ }^{3}$; 0.29 for $V=1$ in. $^{3}$

On figure $2, \chi_{T 0}$ is modified negligibly for $V=$ 0.1 in. $^{3}$

Therefore, $L=450 \mathrm{in} .=37 \mathrm{ft}$ for $V=0.1 \mathrm{in} .^{3}$

Reenter figure 2 for $\left|\tilde{\xi}_{0 L} / \xi_{0}\right|_{0}=0.95, \chi_{T_{0}} / \chi_{T 0}=$ 0.29 to find $\chi_{T 0}=0.5$

Calculate $L$ to be $350 \mathrm{in} .=29 \mathrm{ft}$ for $V=1 \mathrm{in} .^{3}$

b (2). $f=1,000 \mathrm{c} / \mathrm{s}$ :

Using eq 26, $z=610$; therefore, use figures 6 and 7 .

Assume $\chi_{I} / \chi_{T}=49$ (the line volume will probably be small).

Enter figure 6 for $\left|\tilde{\xi}_{\text {oL }} / \xi_{0}\right|=1.05$ to find $\omega L / C=$ 0.031 .

Calculate $L$ from eq 33,26 , and 27 to be 0.066 in.

Calculate $A L$ to be 0.00052 in. $^{3}$

Using eq 34 , it is seen that $\chi_{I 0} / \chi_{T 0}$ is greater than assumed, so that $\omega L / C$, and therefore $L$, is less than the previous estimate. One may note that the estimated length will be so small that the theory essentially predicts that no transmission tubing at all may be used. In fact, the acoustic impedance of the entrance orifice into the pressure instrument or the mechanical impedance of the pressure instrument itself will probably govern the response at this high frequency.

$\mathrm{b}(3) . \quad f=10 \mathrm{c} / \mathrm{s}$ :

Using eq 26, $z=6.1$; therefore, use figures 4 and 5 .

Assume $A L=\infty$, therefore, $\chi_{Y_{0}} / \chi_{T_{0}}=0$.

Enter figure 4 with $\left|\tilde{\xi}_{\text {oL }} / \xi_{0}\right|=1.05$ and $\gamma=1.4$ to find $\chi_{T 0}=0.12$.

Compute $L$ from eq 27 to be 58 in.

Compute $A L$ to be 0.45 in. $^{3}$.

Compute $\chi_{I 0} / \chi_{T 0}$ from eq 28 to be 0.22 for $V=0.1 \mathrm{in}^{3},=2.2$ for $V=1 \mathrm{in}^{3}$

In figure $4, \chi_{T 0}$ is modified to about 0.07 for $V=0.1$ in. $^{8}$

Therefore, $L$ is reduced to about $4 \mathrm{ft}$ for $V=0.1$ in. ${ }^{3}$

Enter figure 4 for $\left|\tilde{\xi}_{O L}\right| \xi_{0} \mid=1.05$, and $\chi_{T_{0}} / \chi_{T 0}=2$ to find $\chi_{T 0}=0.018$.

Compute $L$ to be 22 in. for $V=1$ in. ${ }^{8}$

Compute $A L$ to be 0.17 in. $^{3}$

Compute $\chi_{r_{0}} / \chi_{T_{0}}=6$.

Enter figure 4 to find $\chi_{T 0}=0.007$.
Compute $L$ to be 14 in.

Compute $A L$ to be 0.11 in. $^{3}$

Compute $\chi_{I 0} / \chi_{T 0}=9$.

Enter figure 4 to find $\chi_{T_{0}}=0.004$.

Compute $L$ to be 11 in.

Compute $A L$ to be 0.09 in. $^{3}$

Compute $\chi_{T 0} / \chi_{T 0}=11$.

In figure $4, \chi_{T_{0}}$ is modified negligibly.

Therefore $L=$ about $1 \mathrm{ft}$ for $V=1$ in. ${ }^{8}$

To check the phase angle, enter figure 5 with $\chi_{I 0} / \chi_{T 0}=11$, and $\chi_{T 0}=0.005$, to find $4^{\circ}$.

$\mathrm{b}(4) . f=100 \mathrm{c} / \mathrm{s}$.

$z=61$ (interpolation is necessary).

First estimate from figure 6 and 7.

Assume $\chi_{I} / \chi_{T}=9$.

Enter figure 6 to find $\omega L / C=0.068$

As in b (2), compute $L$ to be 1.5 in.

Compute $A L$ to be 0.011 in.

Compute $\chi_{I} / \chi_{T}=9.1$ for $V=0.1$ in. $^{3} ;=91$ for $V=1$ in. $^{3}$

By figure $6, \omega L / C$ is negligibly modified for $V=0.1$ in. ${ }^{3}$;

Therefore $L=1.5$ in. for $V=0.1 \mathrm{in}^{3}$ is our first estimate.

For $V=1$ in. $^{3}$, we find again that an extremely small tube is predicted, so that the impedance of the entrance orifice will probably govern.

For $V=0.1$ in. ${ }^{3}$ and $L=1.5$ in., estimate $\left[\gamma \chi_{T 0} / 16\right]^{1 / 2}$ to be 01 .

From figure 7 we find that the phase lag is negligible.

Compute $\chi_{T 0}=0.0009$, from (8.2) for $\omega=200 \pi$.

Enter figure 4 for $\chi_{I 0} / \chi_{T 0}=9$ to find $\left|\tilde{\xi}_{O L} / \xi_{0}\right|=1.00$. Interpolating between $\left|\xi_{o L} / \xi_{0}\right|=1$ at $z=6.25$ and $\left|\xi_{\text {oL }} / \xi_{0}\right|=1.05$ at $z=100$ for $z=61$, we find $\left|\tilde{\xi}_{\text {oL }}\right| \xi_{0} \mid$ is negligibly affected.

Therefore $L=1.5 \mathrm{in}$. for $V=0.1 \mathrm{in}^{3}$

\section{Appendix. Development of the Theory}

\section{Introduction}

The difficulties of deriving, elucidating, and comprehending the mathematical results of transmission in tubing from a rigorous point of view, have led the author to treat the problem in a series of somewhat artificial steps. Thus in the previous sections, the elementary solution was presented, to give the reader a general view of the problem, even though many of the details of the solution were slurred over. Here steps are taken, one at a time, to remove the restrictive assump- 
tions made in deriving the elementary solution. Nevertheless, a complete solution to the problem is not obtained. All first-order effects are treated to the point where the solution is correct to frequencies well into the sonic region. However, only an elementary treatment is given for the second-order distortion effects. It is felt that when these second-order effects become appreciable, the solution presented is of no quantitative utility to the instrument system designer or user, but is only indicative as to order of magnitude.

\section{Theory Corrected for Compressibility}

\section{(Infinitesimal Oscillatory Pressures)}

In this approximation, the assumptions are Poiseuille's law of viscous resistance; small fractional pressure excess; and that density, pressure, and viscosity are related by the equation of condition.

For gases one can then write

or

$$
\left.\begin{array}{c}
\frac{\partial p}{\partial x}=-\frac{128}{\pi} \frac{\mu}{D^{4}} Q, \\
\rho \frac{\partial p}{\partial x}=-\frac{128}{\pi} \frac{\mu}{D^{4}} M,
\end{array}\right\}
$$

for Poiseuille's law, and

$$
\frac{\partial M}{\partial x}=-A \frac{\partial p}{\partial t}
$$

for the equation of continuity.

One can eliminate the mass flow $M$, to obtain

$$
\frac{\partial}{\partial x}\left(\frac{\rho}{\mu} \frac{\partial p}{\partial x}\right)=\frac{32}{D^{2}} \frac{\partial \rho}{\partial t} .
$$

By virtue of the assumption of small pressure excess, and the equations of condition (eq 19 and 20 ), we can disregard the differentiation of $\rho / \mu$ in eq. 38 , and replace it by its mean value. Equation 38 then becomes

or

$$
\left.\begin{array}{l}
\frac{\partial^{2} p}{\partial x^{2}}=\frac{32 \mu_{0}}{n p_{0} D^{2}} \frac{\partial p}{\partial t}, \\
\frac{\partial^{2} \xi}{c x^{2}}=\frac{32 \mu_{0}}{n p_{0} D^{2}} \frac{\partial \xi}{\partial t}
\end{array}\right\}
$$

Utilizing the previous definition of $\lambda_{0}$ (eq 10), eq 39 becomes or

$$
\left.\begin{array}{c}
\frac{\partial^{2} \xi}{\partial x^{2}}=\left(\frac{A L}{V} \frac{\lambda_{0}}{n}\right) \frac{1}{L^{2}} \frac{\partial \xi}{\partial t} \\
\frac{\partial^{2} \xi}{\partial x^{2}}=\frac{\lambda_{T 0}}{L^{2}} \frac{\partial \xi}{\partial t}
\end{array}\right\}
$$

where

$$
\lambda_{T 0}=\frac{A L}{V} \frac{\lambda_{0}}{n}
$$

The significance of the new time constant $\lambda_{T 0}$ can be understood by inspection of the definition of $\lambda_{0}$ (eq 10). One may note that $\lambda_{T 0}$ is a time constant based on the tube volume, $A L$, instead of the instrument volume, $V$; and that it gives weight to the exponent of the polytropic process in the tube. It is thus related to the equivalent distributed electrical capacitance of the tube. The weighting by the exponent, $n$, arises from the fact that it represents the additional "stiffness" of the air column in the tube as a polytropic spring.

If, as in the elementary solution, we separate our pressure variable into a space and time part

eq 40 becomes

$$
\xi=\tilde{\xi} e^{j w t},
$$

$$
\text { or } \left.\quad \begin{array}{c}
\frac{d^{2} \tilde{\xi}}{d x^{2}}=\frac{\lambda_{T 0} \omega}{L^{2}} j \tilde{\xi} \\
\frac{d^{2} \tilde{\xi}}{d x^{2}}=\frac{\chi_{T 0}}{L^{2}} j \tilde{\xi}
\end{array}\right\}
$$

where

$$
\chi_{T 0}=\lambda_{T 0} \omega
$$

The quantity $\chi_{T 0}$ is an attenuation factor based on the tube volume.

Equation 42 may be compared with the corresponding equation of the elementary solution, eq 12. It may be noted that it is necessary that $\chi_{T 0}$ be small in order for the elementary solution to be valid.

Referring now to eq 42 , the boundary conditions are

at $x=0$

and at $x=L$

$$
\tilde{\xi}=\xi_{0},
$$

$$
\frac{d \tilde{\xi}}{d x}=-\left(\frac{\lambda_{0} \omega}{m}\right) j \frac{\tilde{\xi}}{L},
$$

(see eq 5, 9, and 24). 
We may redefine a time constant and attenuation factor for the instrument volume, which takes into account the polytropic process as

$$
\left.\begin{array}{c}
\lambda_{I 0}=\frac{\lambda_{0}}{m} \\
\chi_{I 0}=\lambda_{I 0} \omega
\end{array}\right\}
$$

At $x=\mathrm{L}$, eq 44 therefore becomes

$$
\frac{d \tilde{\xi}}{d x}=-\frac{\chi_{I 0}}{L} j \tilde{\xi}
$$

The solution to eq 42 , which satisfies boundary conditions (eq 13 and 46) is

$$
\frac{\tilde{\xi}}{\xi_{0}}=\frac{e^{-\psi_{T 0}}\left(\psi_{T 0}-\psi_{T 0}\right) e^{\psi_{T 0} \frac{x}{L}}+e^{\psi_{T 0}}\left(\psi_{T 0}+\psi_{I 0}\right) e^{-\psi_{T 0} \frac{x}{L}}}{e^{\psi_{T 0}}\left(\psi_{T 0}+\psi_{I 0}\right)+e^{-\psi_{T 0}}\left(\psi_{T 0}-\psi_{T 0}\right)}
$$

where

$$
\left.\begin{array}{l}
\psi_{T 0}=\left[\frac{\chi_{T 0}}{2}\right]^{1 / 2}(1+j) \\
\psi_{T 0}=j \chi_{T 0}
\end{array}\right\}
$$

The new $\psi^{\prime}$ s, which shall be referred to as attenuation parameters, are

$\psi_{T 0}$ an attenuation parameter depending on tube volume;

$\psi_{I 0}$ an attenuation parameter depending on the instrument volume.

The ratio of the fractional pressure excess at the end of the tube $\tilde{\xi}_{L}$ to that at the beginning of the tube $\xi_{0}$ is then

$$
\frac{\tilde{\xi}_{L}}{\xi_{0}}=\frac{\psi_{T 0}}{\psi_{T 0} \cosh \psi_{T 0}+\psi_{I 0} \sinh \psi_{T 0}}
$$

It is instructive to examine the limiting values of this equation. For small $\psi_{T 0}$, the attenuation approaches

$$
\frac{\tilde{\xi}_{L}}{\xi_{0}}=\frac{1}{1+\psi_{I 0}}=\frac{1}{1+j \chi_{I 0}}
$$

the same result as in the elementary theory (see eq 16).

For small values of $\psi_{10}$, the attenuation approaches

$$
\frac{\tilde{\xi}_{L}}{\xi_{0}}=\frac{1}{\cosh \psi_{T 0}}
$$

which for small $\psi_{T 0}$ becomes

$$
\frac{\tilde{\xi}_{L}}{\xi_{0}}=\frac{1}{1+\frac{\psi_{T 0}^{2}}{2}}=\frac{1}{1+\frac{\chi_{T 0}}{2} j} .
$$

The form of eq 50 and 52 is similar. In fact, for small values of both $\psi_{T 0}$ and $\psi_{T 0}$ it is possible to define a composite attenuation factor $\chi$ by the relation

or

$$
\left.\begin{array}{l}
\chi=\chi_{I 0}+\frac{\chi_{T 0}}{[6]^{1 / 2}} \\
\lambda=\lambda_{I 0}+\frac{\lambda_{T 0}}{[6]^{1 / 2}}
\end{array}\right\}
$$

such that the real magnitude of the attenuation is approximately

$$
\left|\frac{\tilde{\xi}_{T}}{\xi_{0}}\right|=\frac{1}{\left[1+\chi^{2}\right]^{1 / 2}}
$$

which preserves the form of the elementary solution.

Equation 18 can be interpreted as meaning that the "proper" time constant of the system can be obtained by adding to the $n$ weighted volume of the instrument, $1 /[6]^{1 / 2}$ of the $m$ weighted volume of the tube, and substituting this in the elementary formula for the time constant of the system.

In principle, for larger values of $\psi_{T 0}$ or $\psi_{T 0}$, a coupling coefficient (of approximately unity) could be introduced as an addition to the coefficient $1 /[6]^{1 / 2}$, which would vary somewhat with the relative magnitude of $\psi_{T 0}$ and $\psi_{I 0}$, to permit strict preservation of the elementary form. It is, however, simpler to compute attenuation from eq 49 .

For liquids, we start from eq 38.

$$
\frac{\partial}{\partial x}\left(\frac{\rho}{\mu} \frac{\partial p}{\partial x}\right)=\frac{32}{D^{2}} \frac{\partial \rho}{\partial t} .
$$

As before, with the aid of eq 22 and 23, we obtain the result

or

$$
\left.\begin{array}{l}
\frac{\partial^{2} \xi}{\partial x^{2}}=\frac{32 \mu_{0} b}{p_{0} D^{2}} \frac{\partial \xi}{\partial t} \\
\frac{\partial^{2} \xi}{\partial x^{2}}=\frac{\lambda_{T 0}}{L^{2}} \frac{\partial \xi}{\partial t} \\
\frac{d^{2} \tilde{\xi}}{d x^{2}}=\frac{\chi_{T 0}}{L^{2}} j \tilde{\xi}
\end{array}\right\}
$$


where

$$
\left.\begin{array}{c}
\lambda_{T 0}=\frac{A L}{V} b \lambda_{0} \\
\chi_{T 0}=\lambda_{T 0} \omega .
\end{array}\right\}
$$

The boundary condition at $x=0$ is

$$
\tilde{\xi}=\xi_{0}
$$

and at $x=L$

$$
\left.\begin{array}{l}
\frac{d \tilde{\xi}}{d x}=-\left(\lambda_{0} b \omega\right) j \frac{\tilde{\xi}}{L} \\
\frac{d \tilde{\xi}}{d x}=-\chi_{I 0} j \frac{\tilde{\xi}}{L}
\end{array}\right\}
$$

where

$$
\left.\begin{array}{l}
\lambda_{I 0}=\lambda_{0} b \\
\chi_{I 0}=\lambda_{I 0} \omega
\end{array}\right\}
$$

(see eq 5, 9, and 25).

The form of eq 54 and 56 is identical with eq 42 and 46 , with the difference that the coefficient in the $\lambda$ 's is the very small compressibility factor rather than the reciprocal of the exponent of the polytropic process. Physically, this simply means that the liquid is a spring of almost infinite stiffness compared to the gas.

Because of the formal identity of the equations, the previous solution holds in toto, with the modified value of $\lambda_{T 0}$. The following interpretation is now possible for the elementary result that there is no attenuation with liquids. The $\lambda_{0}$ time constant of elementary theory did not take into account the effect of liquid compressibility, which is small. If, however, $\lambda_{0}$ is weighted by $b$ (i. e., $\lambda=b \lambda_{0}$ ) then the same attenuation curve holds for both liquids and gases, but with liquids we operate on the very beginning portion of the attenuation curve for gases.

There is one complication that should be considered in liquid tube attenuation. Because of the small compressibility of liquids, it is often possible that the flexibility of the tube gives rise to a compressibility comparable to that of the liquid. The simplest way of taking into account the flexibility of the tube is to define and replace the compressibility factor of the liquid by an effective value $\bar{b}$ and $\bar{k}$.

$$
\left.\begin{array}{l}
\bar{b}=b+\frac{p_{0}-p_{a}}{E} \frac{D}{s} \\
\bar{k}=k+\frac{p_{0}-p_{a}}{p_{0} E} \frac{D}{s}
\end{array}\right\},
$$

where

$p_{0}=$ mean liquid pressure;

$p_{a}=$ ambient external pressure (usually atmospheric);

$E=$ elastic modulus of the tube material;

$s=$ wall thickness of the tube.

In the derivation of eq 58, the assumption has been made that the thickness of the tube wall is small compared to the tube diameter.

\section{Theory Corrected for Finite Oscillatory Pressures}

In this section, we will determine the effect of finite fractional pressure excess on the attenuation in a tube. We assume only that the Poiseuille velocity distribution holds. We will show that the effect of finite pressure excesses is to excite higher harmonics, resulting in a distortion of wave form, and to raise the mean pressure along the tube. The higher harmonics are excited because of the nonlinearity of the equations.

The method of solution selected will be that of expansion in harmonic series in which the excitation of sum frequencies only are considered and the difference frequencies are neglected, so that the solutions obtained are only valid for the leading term of each harmonic. The second order term in the variation of the mean pressure will be estimated separately. We will assume open functions of the distance coordinate for the coefficients of each harmonic term of the series and show that the expansion is valid for moderate values of the initial pressure excess. It is obvious that these distance dependent coefficients must be the solutions of second-order differential equations in order to provide two sets of adjustable constants to satisfy the boundary conditions at the two ends of the tube. However, by considering the solution for an infinite tube (for which only one set of boundary conditions is required) we shall be able to discuss the question of convergence of the solutions.

For the purposes in view, it will turn out to be convenient to derive the equations on a density basis. Density and pressure are, of course, related through the equation of condition. 
For gases, we start from

$$
\frac{\partial}{\partial x}\left(\frac{\rho}{\mu} \frac{\partial p}{\partial x}\right)=\frac{32}{D^{2}} \frac{\partial \rho}{\partial t}
$$

By algebraic manipulation, in which eq 19 and 20 are used to eliminate viscosity and pressure, we obtain

or

$$
\left.\begin{array}{rl}
\frac{\partial}{\partial x}\left(\eta \frac{\partial \eta}{\partial x}\right) & =\frac{\lambda_{T 0}}{L^{2}} \frac{\partial \eta}{\partial t}, \\
\frac{\partial^{2} \eta^{2}}{\partial x^{2}} & =\frac{2 \lambda_{T 0}}{L^{2}} \frac{\partial \eta}{\partial t},
\end{array}\right\}
$$

a nonlinear partial differential equation.

Assume as solution for the density ratio

$$
\eta=1+\eta_{1} e^{j \omega t}+\eta_{2} e^{2 j \omega t}+\eta_{3} e^{3 j \omega t}+\ldots .,
$$

where $\eta_{i}$ is the fractional density amplitude ratio of each harmonic (functions of $x$ ).

At the moment assume that the applied pressure wave has all the mathematical properties necessary to make the Fourier expansion of eq 60 valid. We will discuss this point again.

When eq 60 is substituted in eq 59 and the coefficients of like terms in the respective harmonics equated, the following system of differential equations result for the coefficients $\eta_{i}$.

$$
\left.\begin{array}{c}
1\left(\frac{\psi_{T 0}}{L}\right)^{2} \eta_{1}=\frac{1}{2} \frac{d^{2}}{d x^{2}}\left(\eta_{1} \eta_{0}+\eta_{0} \eta_{1}\right) \\
2\left(\frac{\psi_{T 0}}{L}\right)^{2} \eta_{2}=\frac{1}{2} \frac{d^{2}}{d x^{2}}\left(\eta_{2} \eta_{0}+\eta_{1} \eta_{1}+\eta_{0} \eta_{2}\right) \\
3\left(\frac{\psi_{T 0}}{L}\right)^{2} \eta_{3}=\frac{1}{2} \frac{d^{2}}{d x^{2}}\left(\eta_{3} \eta_{0}+\eta_{2} \eta_{1}+\eta_{1} \eta_{2}+\eta_{0} \eta_{3}\right) \\
\cdot \\
\cdot \\
\dot{\cdot}\left(\frac{\psi_{T 0}}{L}\right)^{2} \eta_{i}= \\
\quad=\frac{1}{2} \frac{d^{2}}{d x^{2}}\left(\eta_{i} \eta_{0}+\eta_{i-1} \eta_{1}+\cdots+\right. \\
\left.\eta_{1} \eta_{t-1}+\eta_{0} \eta_{i}\right)
\end{array}\right\}
$$

The coefficient $\eta_{0}(=1)$ has been added for completeness.

From eq 61 it can be shown that the coefficients $\eta_{i}$ for an infinite tube are equal to

$$
\left.\begin{array}{rl}
\eta_{i}= & \bar{\eta}_{i} e^{-\left(0+[i]^{1 / 2}\right) y}-\frac{\bar{\eta}_{i-1} \bar{\eta}_{1} e^{-\left(1+[i-1]^{1 / 2}\right) y}}{\left(1 \times 0+1 \times 2[i-1]^{1 / 2}\right)}+ \\
& \frac{\bar{\eta}_{i-2} \bar{\eta}_{1}^{2} e^{-\left(2+[i-2]^{1 / 2}\right) y}}{\left(1 \times 0+1 \times 2[i-2]^{1 / 2}\right)\left(2 \times 1+2 \times 2[i-2]^{1 / 2}\right)}- \\
& \frac{\bar{\eta}_{i-3} \bar{\eta}_{1}^{3} e^{-\left(3+[i-3]^{1 / 2}\right) y}}{\left(1 \times 0+1 \times 2[i-3]^{1 / 2}\right)\left(2 \times 1+2 \times 2[i-3]^{1 / 2}\right)\left(3 \times 2+3 \times 2[i-3]^{1 / 2}\right)}+\ldots
\end{array}\right\}
$$


where

$$
y=\psi_{T 0} \frac{x}{L} .
$$

Here $\bar{\eta}_{i}$ is the constant of integration for the complementary solution of each $\eta_{i}$, and $y$ is a dimensionless distance variable.

Substituting the value of the coefficients from eq 62 into eq 60, we obtain the result that, if at the origin $(y=0)$, the density is written in the form $\eta=1+\bar{\eta}_{1} e^{j \omega t}+$

$$
\left.\begin{array}{l}
\left(\bar{\eta}_{2}-\frac{\bar{\eta}_{1} \bar{\eta}_{1}}{1 \times 0+1 \times 2[1]^{1 / 2}}\right) e^{2 j \omega t}+ \\
\left(\bar{\eta}_{3}-\frac{\bar{\eta}_{2} \bar{\eta}_{1}}{1 \times 0+1 \times 2[2]^{1 / 2}}+\right. \\
\left.\frac{\bar{\eta}_{1} \bar{\eta}_{1}^{2}}{\left(1 \times 0+1 \times 2\left[1\left[^{1 / 2}\right)(2 \times 1+2 \times 2[1])^{1 / 2}\right.\right.}\right) e^{3 j \omega t}+\ldots
\end{array}\right\}
$$

at any other point $y$, the density wave will be

$$
\begin{aligned}
\eta= & 1+\bar{\eta}_{1} e^{-\left(0+[1]^{1 / 2}\right) y} e^{j \omega t}+ \\
& \left(\bar{\eta}_{2} e^{-\left(0+[2]^{1 / 2}\right) y}-\frac{\bar{\eta}_{1} \bar{\eta}_{1} e^{-\left(1+[1]^{1 / 2}\right) y}}{1 \times 0+1 \times 2[1]^{1 / 2}}\right) e^{2 j w t}+ \\
& \left(\bar{\eta}_{3} e^{-\left(0+[3]^{1 / 2}\right) y}-\frac{\bar{\eta}_{2} \bar{\eta}_{1} e^{-\left(1+[2]^{1 / 2}\right) y}}{1 \times 0+1 \times 2[2]^{1 / 2}}+\right. \\
& \left.\frac{\bar{\eta}_{1} \bar{\eta}_{1}^{2} e^{-\left(2+[1]^{1 / 2}\right) y}}{\left(1 \times 0+1 \times 2[1]^{1 / 2}\right)\left(2 \times 1+2 \times 2[1]^{1 / 2}\right)}\right) e^{3 j \omega t} \\
& +\ldots
\end{aligned}
$$

Our previous conclusion permits us to assume that expression 64 is manageable (i. e., of limited variation with a time derivative of limited variation) so that it must converge. We may therefore infer the following relations:

For large enough $i$

$$
\left.\begin{array}{c}
0<\left|\frac{\bar{\eta}_{i+1}}{\bar{\eta}_{i}}\right|<1 \\
\left|\frac{\eta_{i+1}}{\eta_{i}}\right| \leqq\left|\frac{\bar{\eta}_{i+1}}{\bar{\eta}_{i}} e^{-\frac{y}{2[i]^{1 / 2}} \mid}\right| \\
0<\left|\frac{\bar{\eta}_{i}}{\bar{\eta}_{1}}\right|<1 \\
\left|\frac{\eta_{i}}{\eta_{1}}\right| \leqq\left|\frac{\bar{\eta}_{i}}{\bar{\eta}_{1}} e^{-y[i]^{1 / 2}}\right| .
\end{array}\right\}
$$

The last line in eq 66 contains our desired conclusion. We can infer from it the maximum number of terms that must be carried along in order to know the distortion to within any desired accuracy. If we assume, for example, that we are interested in only those harmonics whose content at the end of the tube is greater than 1 percent of the applied first harmonic, we can neglect all harmonics greater than the one for which

or

$$
\left|\frac{\bar{\eta}_{i}}{\bar{\eta}_{1}}\right| e^{-\psi_{T_{0}}[i]^{1 / 2}}=0.01
$$

$$
\left.\left|\frac{\bar{\eta}_{i}}{\bar{\eta}_{1}}\right| e^{-\left[\frac{x_{T 0} i}{2}\right]^{1 / 2}}=0.01 .\right\}
$$

For most practical problems, it can be shown that adequate information can be obtained from a knowledge of the first and second harmonic, and rarely, the third harmonic.

To compute the harmonic distortion for a volume terminated tube, we go back to eq 61 . The solution for the density wave becomes

$$
\left.\begin{array}{rl}
\eta= & 1+\left(\bar{\eta}_{1+} e^{y}+\bar{\eta}_{1-} e^{-y}\right) e^{j w t}+ \\
& \left(\bar{\eta}_{2+} e^{[2]^{1 / 2 y}}-\bar{\eta}_{1+}^{2} e^{2 y}+\bar{\eta}_{2-} e^{-[2]^{1 / 2} y}-\right. \\
& \left.\bar{\eta}_{1-}^{2} e^{-2 y}\right) e^{2 j w t}+\left(\bar{\eta}_{3+} e^{[3]^{1 / 2} y}-\right. \\
& \bar{\eta}_{2+} \bar{\eta}_{1+}\left(\frac{3+2[2]^{1 / 2}}{2[2]^{1 / 2}}\right) e^{\left([2]^{1 / 2}+1\right) y}+ \\
& \bar{\eta}_{1+}^{3}\left(\frac{3}{2}\right) e^{3 y}+\bar{\eta}_{3-} e^{-[3]^{1 / 2 y}}- \\
& \bar{\eta}_{2-} \bar{\eta}_{1-}\left(\frac{3+2[2]^{1 / 2}}{2[2]^{1 / 2}}\right) e^{-\left([2]^{1 / 2}+1\right) y}+ \\
& \bar{\eta}_{1-}^{3}\left(\frac{3}{2}\right) e^{-3 y}+\bar{\eta}_{1+} \bar{\eta}_{2-}\left(\frac{3-2[2]^{1 / 2}}{2[2]^{1 / 2}}\right) e^{\left(1-[2]^{1 / 2}\right) y}+ \\
& \bar{\eta}_{2+} \bar{\eta}_{1-}\left(\frac{3-2[2]^{1 / 2}}{2[2]^{1 / 2}}\right) e^{\left(-1+[2]^{1 / 2}\right) y}- \\
& \left.\bar{\eta}_{1+} \bar{\eta}_{1-}^{2}\left(\frac{1}{2}\right) e^{-y}-\bar{\eta}_{1-} \bar{\eta}_{1+}^{2}\left(\frac{1}{2}\right) e^{y}\right) e^{3 j w t}+\cdots
\end{array}\right\}
$$

Here $\bar{\eta}_{i+}, \bar{\eta}_{i-}$ represent the two sets of integration constants necessary to take care of an outgoing and reflected wave in the tube. They are fractional density excesses.

We will consider the boundary conditions to be, for the moment, at $x=0 \quad(y=0)$

$$
\eta=1+\tilde{\eta}_{1} e^{j_{\omega} t}+\tilde{\eta}_{2} e^{j_{\omega} t}+\tilde{\eta}_{3} e^{j \omega t}+\ldots
$$


where $\tilde{\eta}_{i}$ is the amplitude of each input harmonic in the density wave (it differs from $\bar{\eta}_{i}$, which represents both the input harmonic and the excitation amplitudes of that harmonic), and at

$$
\begin{gathered}
x=L\left(y=\psi_{T 0}\right) \\
\eta=1+\left[\tilde{\eta}_{1} \frac{\psi_{T 0}}{\psi_{T 0} \cosh \psi_{T 0}+\psi_{T 0} \sinh \psi_{T 0}}\right] e^{j \omega t}+ \\
{\left[\tilde{\eta}_{2} \frac{[2]^{1 / 2} \psi_{T 0}}{[2]^{1 / 2} \psi_{T 0} \cosh [2]^{1 / 2} \psi_{T 0}+2 \psi_{T 0} \sinh [2]^{1 / 2} \psi_{T 0}}+\right.}
\end{gathered}
$$$$
\eta \frac{\partial \eta}{\partial x}=-\frac{\psi_{I 0}}{\omega L} \frac{\partial \eta}{\partial t}
$$

(see eq 1, 19, 20, and 24).

The application of these two boundary conditions leads to the result that at the end of the tube

$$
\left.\tilde{\eta}_{1}{ }^{2} \frac{[2]^{1 / 2} \psi_{T 0}{ }^{3}\left(\cosh 2 \psi_{T 0}-\cosh [2]^{1 / 2} \psi_{T 0}\right)+2 \psi_{T 0}{ }^{2} \psi_{I 0}\left([2]^{1 / 2} \sinh 2 \psi_{T 0}-\sinh [2]^{1 / 2} \psi_{T 0}\right)+}{[2]^{1 / 2} \psi_{T 0} \psi_{I 0}{ }^{2}\left(\cosh 2 \psi_{T 0}-\cosh [2] \psi_{T 0}\right)}\right] e^{2 j \omega t}+\cdots \cdot
$$

This result is for a given input density wave. It will be shown later that the results shown in eq 71 are only valid when the equation of condition is isothermal (i. e. the "polytropic" coefficient is unity). It therefore follows that if the input

$$
\left.\begin{array}{rl}
\xi= & {\left[\xi_{0} \bar{\psi}_{T 0} \frac{\psi_{T 0}}{\cosh \psi_{T 0}+\psi_{I 0} \sinh \psi_{T 0}}\right] e^{j^{\omega t}}+\frac{[2]^{1 / 2} \psi_{T 0}}{[2]^{1 / 2} \psi_{T 0} \cosh [2]^{1 / 2} \psi_{T 0}+2 \psi_{I 0} \sinh [2]^{1 / 2} \psi_{T 0}}} \\
& {\left[\begin{array}{c}
\xi_{1}+\xi_{0}^{2} \\
\left.\begin{array}{c}
\psi_{T 0}^{2}\left(\cosh 2 \psi_{T 0}-\cosh [2]^{1 / 2} \psi_{T 0}\right)+[2]^{1 / 2} \psi_{T 0} \psi_{T 0}\left([2]^{1 / 2} \sinh 2 \psi_{T 0}-\right. \\
\left.\sinh [2]^{1 / 2} \psi_{T 0}\right)+\psi_{I 0}^{2}\left(\cosh 2 \psi_{T 0}-\cosh [2]^{1 / 2} \psi_{T 0}\right)
\end{array}\right]
\end{array}\right] e^{j \omega t}+\cdots}
\end{array}\right\}
$$

For liquids, we can start from eq 38

$$
\frac{\partial}{\partial x}\left(\frac{\rho}{\mu} \frac{\partial p}{\partial x}\right)=\frac{32}{D^{2}} \frac{\partial \rho}{\partial t}
$$

By the use of eq 22 and 23, we obtain

$$
\frac{\partial^{2} \eta^{2}}{\partial x^{2}}=\frac{2 \lambda_{T 0}}{L^{2}} \frac{\partial \eta}{\partial t}
$$

where

$$
\lambda_{T o}=\frac{A L}{V} b \lambda_{0}
$$

The equation is exactly the same as before with the single modification that $1 / b$ is substituted for $n$ and $m$, so that our previous result (eq 73) holds.

The change in mean density along the tube can be estimated from eq 59 and 70 . The equation of motion (eq 59) requires that the second derivative of the mean square density vanishes, or that the first derivative is constant. However, the end boundary condition (eq 70) requires that the first derivative of the mean square density vanishes at the end of the tube, and therefore along the entire tube, so that the mean square density and therefore the mean square pressure must remain constant along the tube. The leading part of the second order change in mean pressure arises, therefore, from the steady state portion of the square of the amplitude of the fundamental. It can be simply shown that the increase in mean pressure at the instrument is given by

$$
\xi_{0} \Delta p\left[1+\left|\tilde{\xi}_{\text {OL }} / \xi_{0}\right|^{2}\right] / 4
$$

\section{Theory Corrected for Acceleration}

In this section, we will remove the main restrictive assumption - the assumed Poiseuille velocity distribution. In order to do this, it is necessary to go back to the equations of hydrodynamics. Since the complete theory is too extensive to be treated in this paper, we will simply state the results.

It is possible to take the Navier-Stokes equations of hydrodynamics (the equations of motion), combine them with the equation of continuity, and with the energy equation, which represents a detailed energy balance among thermal and kinetic energies, to arrive at the Kirchoff equations of sound. (See Rayleigh, Theory of sound, volume 2, article 348.) These equations are valid to first order. This procedure was followed, making no assumption as to the form of equation of state for the fluid, and the following results were obtained for the attenuation parameter, and the velocity in an infinite tube: 


$$
\left(\frac{\psi_{T}}{L}\right)^{2}=-\frac{\omega^{2}}{C^{2}} \frac{1+\frac{2(\gamma-1) J_{1}\left(g \frac{D}{2}\right)}{g \frac{D}{2} J_{0}\left(g \frac{D}{2}\right)}}{1-\frac{2 J_{1}\left(h \frac{D}{2}\right)}{h \frac{D}{2} J_{0}\left(h \frac{D}{2}\right)}}
$$

where

$$
\begin{aligned}
& g \frac{D}{2}=(1-j) \frac{D}{2}\left[\frac{\omega \sigma_{0}}{2 \nu_{0}}\right]^{1 / 2} \\
& h \frac{D}{2}=(1-j) \frac{D}{2}\left[\frac{\omega}{2 \nu_{0}}\right]^{1 / 2}
\end{aligned}
$$

and

$$
Q=-\frac{\pi D^{2}}{4 j \omega \rho_{0}}\left(1-\frac{2 J_{1}\left(h \frac{D}{2}\right)}{h \frac{D}{2} J_{0}\left(h \frac{D}{2}\right)}\right) \frac{\partial p}{\partial x},
$$

where

$C$ is the Laplacian velocity of sound in the fluid;

$g$ and $h$ are arguments of the Bessel functions for unit tube radius;

$J_{0}$ and $J_{1}$ are the zero ${ }^{\text {th }}$ and first order Bessel functions;

$\sigma_{0}$ is the mean Prandtl number of the fluid

$$
\left(=\frac{c_{p_{0} \mu_{0}}}{K_{0}}\right)
$$

$K_{0}$ is the mean thermal conductivity of the fluid;

$c_{p_{0}}$ is the mean specific heat at constant pressure of the fluid;

$\nu_{0}$ is the kinematic viscosity of the fluid $\left(=\frac{\mu_{0}}{\rho_{0}}\right)$.
The attenuation parameter in eq 74 is to be interpreted as before (see eq 47) as the exponent in the form $e^{ \pm \psi_{T} \frac{x}{L}}$.

Equal 64 and 65 are of doubtful value for

$$
\frac{\nu_{0}}{C D}>1
$$

or

$$
\frac{v_{0} \omega}{C^{2}}>1
$$

These restrictions are violated at high vacuum or very high frequencies.

It is instructive to evaluate eq 74 and 75 for small values of the Bessel function arguments. They become

$$
\left.\begin{array}{rl}
\left(\frac{\psi_{T}}{L}\right)^{2} & =\frac{32 j \omega \nu_{0} \gamma}{C^{2} D^{2}} \\
Q & =-\frac{\pi D^{4}}{128 \mu_{0}} \frac{\partial p}{\partial x}
\end{array}\right\}
$$

which are precisely the results assumed in eq 1 and 42 under the condition in eq 42 that the "polytropic" coefficient is unity. This arises because the value $C^{2} / \gamma$ in eq 77 is the square of the Newtonian velocity of sound, which for gases is $p_{0} / \rho_{0}$. Eq 74 and 75 , which take into account the heat conduction, thus demonstrate that, when the previous results are valid, the equation of condition is the isothermal. At higher frequencies the modifying term in eq 74 may be regarded as the "polytropic" coefficient. To bring this out explicitly, eq 74 and 75 may be written as

$$
\left(\frac{\psi_{T}}{L}\right)^{2}=\frac{32 j \omega \nu_{0} \gamma}{C^{2} D^{2}}\left[\frac{\frac{\left(h \frac{D}{2}\right)^{2}}{8}}{\frac{2 J_{1}\left(h \frac{D}{2}\right)}{h \frac{D}{2} J_{0}\left(h \frac{D}{2}\right)}-1}\right]\left(\frac{1+\frac{2(\gamma-1) J_{1}\left(g \frac{D}{2}\right)}{g \frac{D}{2} J_{0}\left(g \frac{D}{2}\right)}}{\gamma}\right) Q=-\frac{\pi D^{4}}{128 \mu_{0}} \frac{\partial p}{\partial x}\left(\frac{\frac{2 J_{1}\left(h \frac{D}{2}\right)}{h \frac{D}{2} J_{0}\left(h \frac{D}{2}\right)}}{\frac{\left(h \frac{D}{2}\right)^{2}}{8}}\right)
$$

We may regard eq 78 as an extended definition of the attenuation parameter $\psi_{T}$, and as the modified velocity that replaces Poiseuilles law. It is therefore used without the zero subscript, which is used to denote the Poiseuille regime.

If we now bring in the end boundary condition, namely

$$
Q=\frac{V}{m p_{0}} \frac{\partial p}{\partial t}
$$

for a gas, or

$$
Q=\frac{V b}{p_{0}} \frac{\partial p}{\partial t}
$$


for a liquid, we obtain

$$
\frac{d \tilde{\xi}}{d x}=-\frac{128 \mu_{0} V j \omega}{\pi D^{4} m p_{0}} \tilde{\xi}\left(\frac{\frac{\left(h \frac{D}{2}\right)^{2}}{8}}{\frac{2 J_{1}\left(h \frac{D}{2}\right)}{h \frac{D}{2} J_{0}\left(h \frac{D}{2}\right)}-1}\right)
$$

for a gas, or

$$
\frac{d \tilde{\xi}}{d x}=-\frac{128 \mu_{0} V j \omega b}{\pi D^{4} p_{0}} \tilde{\xi}\left(\frac{\frac{\left(h \frac{D}{2}\right)^{2}}{8}}{\frac{2 J_{1}\left(h \frac{D}{2}\right)}{h \frac{D}{2} J_{0}\left(h \frac{D}{2}\right)}-1}\right)
$$

for a liquid.

Let

$$
\chi_{I}=\frac{128 \mu_{0} V \omega L}{\pi D^{4} m p_{0}}\left(\frac{\frac{\left(h \frac{D}{2}\right)^{2}}{8}}{\frac{2 J_{1}\left(h \frac{D}{2}\right)}{h \frac{D}{2} J_{0}\left(h \frac{D}{2}\right)}-1}\right)
$$

for a gas, or

$$
\chi_{I}=\frac{128 \mu_{0} V^{\top} \omega L b}{\pi D^{4} p_{0}}\left(\frac{\frac{\left(h \frac{D}{2}\right)^{2}}{8}}{\frac{2 J_{1}\left(h \frac{D}{2}\right)}{h \frac{D}{2} J_{0}\left(h \frac{D}{2}\right)}-1}\right)
$$

for a liquid.

We have thus corresponding extensions of our definition of $\chi_{I}$ to cover all frequencies. The limiting value of $\chi_{I}$ for small arguments obviously becomes the previous value for $\chi_{I 0}$.

Since the only modification has been to extend the definitions of $\psi_{T}$ and $\psi_{I}$ without changing the form of the equations to be solved (namely eq 42 and 46), the previous result (eq 49) is strictly valid. The results however are now correct for frequencies well into the audio range.

It is not possible to use the results of this section to extend the range of validity of the calculated distortion for finite pressure amplitudes. To do this rigorously would require going back to the second-order terms neglected in Kirchoff's equations, which is an extremely arduous procedure.
It must therefore be concluded that the distortion calculated in section (3) is valid whenever the Poiseuille regime holds, which also means that the "polytropic" coefficient in the distortion must be taken as unity. The distortion may be validly calculated from eq 73 when

$$
\frac{\omega D^{2}}{4 \nu_{0}}<1,
$$

and when the applied pressure amplitude is sufficiently small at the applied frequency (sufficiently small enough Reynolds number) to permit laminar flow.

\section{Theory Corrected for Finite Length-End Effects}

There is one additional factor that must be considered for completeness - the end effect. An estimate of its magnitude will be made for the Poiseuille regime. It arises from the fact that it takes an appreciable length of tubing to set up the Poiseuille velocity distribution in the transmission tube. The character of the entrance flow is that the axial velocity is flat at the entrance, gradually developing an approximately parabolic (laminar) boundary with a core of uniform velocity, until the approximately parabolic distribution fills the tube. It is evident that boundary layer theory may be used, and for our purposes an extremely crude boundary layer theory.

We go back to the equations of hydrodynamics and make the following assumptions: (1) that the entrance flow is incompressible, (2) that the variation of pressure in the radial direction is negligible in the entrance portion, (3) that quadratic terms in velocity are negligible in the boundary layer, (4) that the core of the velocity distribution is potential.

For our purposes we need only write the equations of motion for the potential core as

$$
\frac{\partial u_{p}}{\partial t}+u_{p} \frac{\partial u_{p}}{\partial x}=-\frac{1}{\rho_{0}} \frac{\partial p}{\partial x},
$$

where $u_{p}$ is the axial velocity in the potential core.

Let

$$
u_{p}=\frac{\partial \phi}{\partial x}
$$

where $\phi$ is the velocity potential

then

$$
\frac{\partial}{\partial x}\left(\frac{\partial \phi}{\partial t}\right)+\frac{1}{2} \frac{\partial u_{p}^{2}}{\partial x}+\frac{1}{\rho_{0}} \frac{\partial p}{\partial x}=0
$$

or

$$
\frac{\partial \phi}{\partial t}+\frac{1}{2} u_{p}^{2}+\frac{p}{\rho_{0}}=f(t)
$$


where $f(t)$ is an arbitrary function of time.

$\mathrm{Eq}$ (86) represents the Bernoulli integral. It can be written in the form

$$
p(x, t)+\frac{1}{2} \rho_{0} u_{p}^{2}=f(t)-\frac{\partial \phi(x, t)}{\partial t} .
$$

The boundary conditions are a prescribed pressure variation at $x=0$, the entrance, with a flat velocity profile $\left(\bar{u}_{P}=\bar{u}\right.$ where $\bar{u}$ is the average velocity across the section) and a parabolic distribution of velocity át some point $x=l$ downstream $\left(\frac{u_{p}}{2}=\bar{u}\right)$. The assumption of incompressible flow makes $\bar{u}$ the same at both sections. these conditions lead to the result that

$$
p(0, t)=p(l, t)+\frac{3}{2} \rho_{0} \bar{u}^{2}+\frac{\partial \phi}{\partial t}(l, t)-\frac{\partial \phi(0, t)}{\partial t} .
$$

If we now refer to the arguments given in Goldstein "Modern Developments in Fluid Mechanics," vol. 1, pp 299 to 308 for the static case, we find on p. 302 that

$p(0)=p(l)+\frac{3}{2} \rho_{0} \bar{u}^{2}+\frac{16 \rho_{0} \bar{u}^{2}}{\frac{D}{2} R_{e}}\left(l-.0575 \frac{D}{2} R_{e}\right)$,

where $R_{e}$ is the Reynolds number.

It follows from these two equations that the leading term for the entrance loss in the oscillatory case is the usual $\rho_{0} \bar{u}^{2}$ loss.

We may therefore adopt the exact static result (see p. 308 of Goldstein) that

$$
p(0, t)=p(l, t)+2.41\left(\frac{1}{2} \rho_{0} \bar{u}^{2}\right)+\frac{16 x}{\frac{D}{2} R_{e}} \rho_{0} \bar{u}^{2},
$$

where the last term represents the Poiseuille viscous resistance.

Therefore the effect of the entrance is to cause a pressure drop given by

$$
\Delta p=\rho_{0} \bar{u}^{2},
$$

the exact coefficient 1.2 being unimportant for our purposes. We will regard eq 91, not as being exact, but as indicating the order of magnitude of the entrance correction.

If we substitute the Poiseuille velocity into eq (91) and evaluate the pressure gradient of the Poiseuille distribution from eq (47), we arrive at the result that the pressure excess just inside the tube $\xi^{1}$ is approximately given by

$\xi^{1}=\xi_{0} e^{j \omega t}-\frac{j D^{2} \omega}{32 \nu_{0}}\left(\frac{\psi_{T 0} \tanh \psi_{T 0}+\psi_{I 0}}{\psi_{T 0}+\psi_{I 0} \tanh \psi_{T 0}}\right)^{2} \xi_{0}^{2} e^{2 j \omega t}$.

Equation 92 is the desired result. It shows that the approximate effect of the entrance is to distort each input harmonic. It can be interpreted as meaning that the effect of the entrance is the same as if it did not exist, but with the fundamental harmonic generator replaced by a fundamental and a second harmonic generator. The firstorder terms are thus left unaffected, and the only equation requiring modification is the attenuated second harmonic.

If eq 92 is used as the input pressure for a pure sinusoidal input in the fluid conduit, eq 73 becomes

$$
\left.\begin{array}{rl}
\xi=\xi_{0} & {\left[\frac{\psi_{T 0}}{\psi_{T 0} \cosh \psi_{T 0}+\psi_{T 0} \sinh \psi_{T 0}}\right] e^{j \omega t}+\frac{[2]^{1 / 2} \psi_{T 0} \xi_{0}^{2}}{[2]^{1 / 2} \psi_{T 0} \cosh [2]^{1 / 2} \psi_{T 0}+2 \psi_{T 0} \sinh [2]^{1 / 2} \psi_{T 0}} e^{2 j \omega t} \times} \\
& {\left[\begin{array}{l}
\psi_{T 0}^{2}\left(\left[1+N^{2}\right] \cosh 2 \psi_{T 0}-N^{2}-\cosh [2]^{1 / 2} \psi_{T 0}\right)+[2]^{1 / 2} \psi_{T 0} \psi_{T 0}\left([2]^{1 / 2}\left[1-N^{2}\right] \sinh 2 \psi_{T 0}-\right. \\
\left.\sinh [2]^{1 / 2} \psi_{T 0}\right)+\psi_{T 0}^{2}\left(\left[1-N^{2}\right] \cosh 2 \psi_{T 0}-N^{2}-\cosh [2]^{1 / 2} \psi_{T 0}\right)
\end{array}\right]}
\end{array}\right\}
$$

where

$$
N^{2}=\frac{j D^{2} \omega}{32 \nu_{0}}=-\frac{\left(h \frac{D}{2}\right)^{2}}{8} .
$$

$N$ is a dimensionless parameter.

The second term in eq 93 gives the second harmonic distortion.

Actually from the condition under which eq 93 is valid (namely eq 83), the value of $N^{2}$ must be small, so that it is a matter of indifference whether it is used in eq 93 or not, and we will therefore neglect it.

\section{Summary}

There only remains the task of recapitulating the pertinent results and presenting them for 
computational convenience. To accomplish this some minor notational changes will be made.

In eq 49 , it was shown that the complex attenuation of the fundamental is given by

$$
\frac{\tilde{\xi}_{O L}}{\xi_{0}}=\frac{\psi_{T}}{\psi_{T} \cosh \psi_{T}+\psi_{L} \sinh \psi_{T}} .
$$

where $\xi_{O L}$ is the complex amplitude of the frac- tional pressure excess of the fundamental at the end of the tube, and the subscript $O$ refers to the fundamental.

In eq 93 , it was shown that the complex amplitude of the second harmonic distortion due to a pure sinusoidal pressure input is given in the form of its ratio to the input amplitude of the fundamental by

$$
\left(\frac{\tilde{\xi}_{I L}}{\xi_{0}}\right)_{0}=\frac{\left[\begin{array}{l}
\xi_{0} \psi_{T 0}\left[\psi_{T 0}^{2}\left(\cosh 2 \psi_{T 0}-\cosh [2]^{1 / 2} \psi_{T 0}\right)+[2]^{1 / 2} \psi_{T 0} \psi_{T 0}\left([2]^{1 / 2} \sinh 2 \psi_{T 0}-\right.\right. \\
\left.\left.\sinh [2]^{1 / 2} \psi_{T 0}\right)+\psi_{T 0}^{2}\left(\cosh 2 \psi_{T 0}-\cosh [2]^{1 / 2} \psi_{T 0}\right)\right]
\end{array}\right]}{[2]^{1 / 2}\left[\psi_{T 0} \cosh \psi_{T 0}+\psi_{T 0} \sinh \psi_{T 0}\right]^{2}\left[[2]^{1 / 2} \psi_{T 0} \cosh [2]^{1 / 2} \psi_{T 0}+2 \psi_{T 0} \sinh [2]^{1 / 2} \psi_{T 0}\right]},
$$

where $\tilde{\xi}_{I L}$ is the complex amplitude of the fractional pressure excess of the second harmonic at the end of the tube; $\psi_{T 0}$ and $\psi_{I 0}$ are the values of the attenuation parameter computed on the basis of the Poiseuille velocity distribution.

In eq 76 , it was shown that eq 95 is valid if

$$
\left.\begin{array}{l}
\frac{\nu_{0}}{C D}<1 \\
\frac{\nu_{0} \omega}{C^{2}}<1
\end{array}\right\}
$$

In eq 83 , it was shown that eq 96 is valid if

$$
\frac{\omega D^{2}}{4 \nu_{0}}<1
$$

In eq 48 , the attenuation parameters were defined as

$$
\left.\begin{array}{c}
\psi_{T}^{2}=j \chi_{T} \\
\psi_{I}=j \chi_{I}
\end{array}\right\}
$$

In eq 78,81 , and 82 , with slight modifications for generality, the attenuation factors were determined to be most generally

$$
\left.\begin{array}{rl}
\chi_{T}=\frac{32 \nu_{0} \omega}{C_{T}^{2}}\left(\frac{L}{D}\right)^{2}\left(\frac{2(\gamma-1) J_{1}\left(g \frac{D}{2}\right)}{g \frac{D}{2} J_{0}\left(g \frac{D}{2}\right)}\right. & \gamma \\
\gamma & \left.\frac{\frac{\left(h \frac{D}{2}\right)^{2}}{8}}{\frac{2 J_{1}\left(h \frac{D}{2}\right)}{h \frac{D}{2} J_{0}\left(h \frac{D}{2}\right)}-1}\right] \times \\
\chi_{1}=\frac{32 \nu_{0} \omega}{C_{I}^{2}}\left(\frac{L}{D}\right)^{2} \frac{V}{A L} & \frac{\left(h \frac{D}{2}\right)^{2}}{\frac{8}{2 J_{1}\left(h \frac{D}{2}\right)}}
\end{array}\right\}
$$

while the attenuation factors for Poiseuille flow are

$$
\left.\begin{array}{l}
\chi_{T 0}=\frac{32 \nu_{0} \omega}{C_{T}^{2}}\left(\frac{L}{D}\right)^{2} \\
\chi_{I 0}=\frac{32 v_{0} \omega}{C_{1}^{2}}\left(\frac{L}{D}\right)^{2} \frac{V}{A L}
\end{array}\right\}
$$




$$
\left.\begin{array}{l}
g \frac{D}{2}=(1-j) \frac{D}{2}\left[\frac{\omega \sigma_{0}}{2 \nu_{0}}\right]^{1 / 2} \\
h \frac{D}{2}=(1-j) \frac{D}{2}\left[\frac{\omega}{2 \nu_{0}}\right]^{1 / 2}
\end{array}\right\}
$$

Here $C_{T}$ is the velocity of sound appropriate to the tube;

$C_{I}$ is the velocity of sound appropriate to the instrument volume.

For computational purposes, the attenuation factors can be made less complicated by the introduction of two new functions.

Let

$$
\left(\frac{1+\frac{2(\gamma-1) J_{1}\left(g \frac{D}{2}\right)}{g \frac{D}{2} J_{0}\left(g \frac{D}{2}\right)}}{\gamma}\right)\left(\frac{\frac{\left(h \frac{D}{2}\right)^{2}}{8}}{\frac{2 J_{1}\left(h \frac{D}{2}\right)}{h \frac{D}{2} J_{0}\left(h \frac{D}{2}\right)}-1}\right)
$$

and

$$
F_{2}=\left(\frac{\gamma}{1+(\gamma-1) \frac{2 J_{1}\left(g \frac{D}{2}\right)}{g \frac{D}{2} J_{0}\left(g \frac{D}{2}\right)}}\right)
$$

where $F_{1}$ and $F_{2}$ are correction functions to the Poiseuille attenuation factors.

The attenuation factors then become

$$
\begin{aligned}
\chi_{T} & =\frac{32 \nu_{0} \omega}{C_{T}{ }^{2}}\left(\frac{L}{D}\right)^{2} F_{1} \\
\chi_{I} & =\frac{32 \nu_{0} \omega}{C_{I}^{2}}\left(\frac{L}{D}\right)^{2}\left(\frac{V}{A L}\right) F_{1} F_{2} .
\end{aligned}
$$

In order to obtain consistency with our previous results, we introduce the following definitions:

\section{For a gas:}

It can be shown both from kinetic theory and from experimental data that the value of the Prandtl number for a gas is approximately unity. Differences from unity are unimportant for our purposes Therefore $g$ and $h$ in eq (102) may be regarded as equal. We may therefore define $F_{1}$ and $F_{2}$ as

$$
F_{1}\left(h \frac{D}{2}, \gamma\right)=\left(\frac{2(\gamma-1) J_{1}\left(h \frac{D}{2}\right)}{h \frac{D}{2} J_{0}\left(h \frac{D}{2}\right)}\right)\left(\frac{\frac{\left(h \frac{D}{2}\right)^{2}}{8}}{\gamma}\right)\left(\frac{2 J_{1}\left(h \frac{D}{2}\right)}{h \frac{D}{2} J_{0}\left(h \frac{D}{2}\right)}-1\right)
$$

$$
F_{2}\left(h \frac{D}{2}, \gamma\right)=\left(\frac{\gamma}{1+(\gamma-1) \frac{2 J_{1}\left(h \frac{D}{2}\right)}{h \frac{D}{2} J_{0}\left(h \frac{D}{2}\right)}}\right)
$$

These functions therefore depend only on two variables, instead of three as in eq (103).

Equation 78 shows that the velocity of sound appropriate to the tube for eq 104 is the Newtonian velocity, which for a gas is $\left[p_{0} / \rho_{0}\right]^{1 / 2}$. Equation 81 indicates that the velocity of sound appropriate to the instrument volume for eq 104 is the "polytropic" velocity or $\left[m p_{0} / \rho_{0}\right]^{1 / 2}$. Therefore for a gas, the attenuation factors may be computed from

$$
\begin{aligned}
\chi_{T} & =\frac{32 \mu_{0} \omega}{p_{0}}\left(\frac{L}{D}\right)^{2} F_{1}\left(h \frac{D}{2}, \gamma\right) \\
\chi_{I} & =\frac{32 \mu_{0} \omega}{m p_{0}}\left(\frac{L}{D}\right)^{2}\left(\frac{V}{A L}\right) F_{1}\left(h \frac{D}{2}, \gamma\right) F_{2}\left(h \frac{D}{2}, \gamma\right)
\end{aligned}
$$

where $F_{1}, F_{2}$, and $h \frac{D}{2}$ may be computed from eq 102 and 105 .

It is convenient to utilize one more variable, the ratio $\chi_{I} / \chi_{T}$, which from eq 106 has the value

$$
\frac{\chi_{I}}{\chi_{T}}=\frac{1}{m} \frac{V}{A L} F_{2}\left(h \frac{D}{2}, \gamma\right)
$$

\section{For a liquid:}

It has been stated that $\gamma$ can be satisfactorily taken as unity for a liquid. This similarly makes the functions $\mathbf{F}_{1}$ and $\mathbf{F}_{2}$ (see eq. 103) independent of $g$ (or really of the Prandtl number). In that case, the definition of $F_{1}$ and $F_{2}$ for a gas (eq. 105) holds for a liquid, if $\gamma$ is taken as unity. Continuity of definition is thus provided for both liquid and gas attenuation.

Equation 78 shows that the velocity of sound appropriate to the tube for eq 104 is the Newtonian velocity of sound. However, consistent with eq. 
55 and 58 , the Newtonian velocity must be based on the effective compressibility of the liquid and tube. From eq 82 it is seen that the velocity of sound appropriate to the instrument volume, however, is based on the real compressibility of the fluid (the difference between adiabatic, "polytropic," and isothermal compressibilities is assumed negligible). It has been assumed that the compressibility of the instrument volume is included in the definition of the effective instrument volume. It follows therefore that the attenuation factors for a liquid can be computed from

$$
\left.\begin{array}{l}
\chi_{T}=32 \mu_{0} k_{0} \omega\left(\frac{L}{D}\right)^{2}\left(1+\frac{p_{0}-p_{a}}{p_{0}} \frac{1}{\mathrm{k}_{0} E} \frac{D}{S}\right) F_{1}\left(h \frac{D}{2}, 1\right) \\
\chi_{I}=32 \mu_{0} k_{0} \omega\left(\frac{L}{D}\right)^{2} \frac{V}{A L} F_{1}\left(h \frac{D}{2}, 1\right) F_{2}\left(h \frac{D}{2}, 1\right) \\
\frac{\chi_{T}}{\chi_{I}}=\frac{V}{A L}\left(\frac{1}{1+\frac{p_{0}-p_{a}}{p_{0}} \frac{1}{k_{0} E} \frac{D}{S}}\right) F_{2}\left(h \frac{D}{2}, 1\right)
\end{array}\right\}
$$

and

$$
\left.\begin{array}{l}
\chi_{T 0}=32 \mu_{0} k_{0} \omega\left(\frac{L}{D}\right)^{2}\left(1+\frac{p_{0}-p_{a}}{p_{0}} \frac{1}{k_{0} E} \frac{D}{S}\right) \\
\chi_{I 0}=32 \mu_{0} k_{0} \omega\left(\frac{L}{D}\right)^{2} \frac{V}{A L}
\end{array}\right\}
$$

It is now possible to compute the real attenuation and lagging phase angle for the first and second harmonics. If the attenuation parameters in eq 95 are regarded as the low frequency parameters based on the Poiseuille distribution (i. e. the ones with zero subscripts), then the real attenuation and lagging phase angle can be computed from

$$
\left.\begin{array}{l}
\mid \begin{array}{c}
\left.\frac{\xi_{O L}}{\xi_{0}}\right|_{0}=\left[\begin{array}{c}
\frac{2}{\cosh \left[2 \chi_{T 0}\right]^{1 / 2}+\cos \left[2 \chi_{T 0}\right]^{1 / 2}+\left(\frac{\chi_{T 0}}{\chi_{T 0}}\right)\left[2 \chi_{T 0}\right]^{1 / 2}\left(\sinh \left[2 \chi_{T 0}\right]^{1 / 2}-\right.} \\
\left.\sin \left[2 \chi_{T 0}\right]^{1 / 2}\right)+\left(\frac{\chi_{I 0}}{\chi_{T 0}}\right)^{2} \chi_{T 0}\left(\cosh \left[2 \chi_{T 0}\right]^{1 / 2}-\cos \left[\chi_{T 0}\right]^{1 / 2}\right)
\end{array}\right] \\
\tanh \left[\frac{\chi_{T 0}}{2}\right]^{1 / 2} \tan \left[\frac{\chi_{T 0}}{2}\right]^{1 / 2}+\left(\frac{\chi_{I 0}}{\chi_{T 0}}\right)\left[\frac{\chi_{T 0}}{2}\right]^{1 / 2}\left(\tanh \left[\frac{\chi_{T 0}}{2}\right]^{1 / 2}+\tan \left[\frac{\chi_{T 0}}{2}\right]^{1 / 2}\right)\left[\frac{\chi_{T 0}}{2}\right]^{1 / 2}\left(\tanh \left[\frac{\chi_{T 0}}{2}\right]^{1 / 2}-\tan \left[\frac{\chi_{T 0}}{2}\right]^{1 / 2}\right)
\end{array} \\
\text { subscript means that these are the } \\
\frac{2 J_{1}(y)}{y J_{0}(y)}=\frac{1}{y^{2}}-\frac{2 j}{y}
\end{array}\right\}
$$
values for the Poiseuille flow regime. Graphs of these equations are quite useful for computing attenuation. Since $\chi_{T 0}$ is proportional to frequency (see eq 101), while $\chi_{I 0} / \chi_{T 0}$ is proportional to the ratio of instrument volume to line volume (see eq 107), a family of curves of attenuation or phase angle plotted against $\chi_{T 0}$ for different values of $\chi_{T_{0}} / \chi_{T 0}$ are frequency response curves for different volume ratios. These curves are presented as figures 2 and 3 .

At higher frequencies, where the functions $F_{1}$ and $F_{2}$ take on values appreciably different from unity, the expressions become extremely complicated. It is therefore of utility to examine their high frequency behavior.

At high frequency, we will use the approximation

If we define a new parameter $z\left(\right.$ related to $\left.h \frac{D}{2}\right)$, which characterizes the fluid regime, as

$$
z=\frac{D^{2} \omega}{4 \nu_{0}},
$$

a frequency parameter $\omega L / C$, where

$$
\frac{\omega L}{C}=\left[\frac{\chi_{T 0} z}{8 \gamma}\right]^{1 / 2},
$$

and a volume ratio $R$ defined as

$$
R=\gamma \frac{\chi_{T 0}}{\chi_{T 0}}
$$

it can be shown that at high frequency 


$$
\begin{aligned}
\left|\frac{\tilde{\xi}_{\text {OL }}}{\xi_{0}}\right| & =\left[\frac{2}{1+\cos \frac{2 \omega L}{C}-R\left(\frac{2 \omega L}{C}\right) \sin \frac{2 \omega L}{C}+\frac{R^{2}}{4}\left(\frac{2 \omega L}{C}\right)^{2}\left(1-\cos \frac{2 \omega L}{C}\right)}\right]^{1 / 2} \\
\tan \delta_{O} & =\left[\frac{\gamma \chi_{T 0}}{16}\right]^{1 / 2} \frac{\left(1+R \frac{2-\gamma}{\gamma}\right) \tan \frac{\omega L}{C}+R \frac{\omega L}{C}}{1-R \frac{\omega L}{C} \tan \frac{\omega L}{C}} .
\end{aligned}
$$

This is the solution for the undamped acoustic resonance of a tube and instrument.

In computing these quantities in eq 115, it is assumed that $\chi_{T 0}$ is smaller than $\chi_{T 0} z_{a}$, or that $\chi_{T 0}$ is small compared to $\omega L / C$. In the solution for the undamped case, the phase angle lag is usually regarded as zero up to the first resonance. However, the given expression permits first-order computation of the phase angle lag valid for values of $\chi_{T 0}$ small compared to $\omega L / C$, even though the overshoot is given as undamped. Practically, this means for values of $\left[\frac{\gamma \chi_{T 0}}{16}\right]^{1 / 2}$ of the order of one or less.

These quantities are presented in figures 6 and 7. It can be shown that they are valid for values of $z \geq 100$, whereas the low frequency curves (figs. 2 and 3 ) are valid for $z \leq 1$ (see eq 98). We will state without proof that the parameter $z$, which characterizes the flow regime, is closely related to a damping coefficient. Figures 2 and 3 will therefore be referred to as the large damping curves, and figures 6 and 7 will be referred to as the undamped curves.

Unfortunately, in many instances, a knowledge of the highly damped behavior (figs. 2 and 3) and the undamped behavior is not sufficient. Curves have therefore been drawn for a value of $z$ about "half-way" between 1 and 100, namely $z=6.25$ (see figs. 4 and 5). In order to preserve a scale proportional to frequency, the quantity $\chi_{T 0}$ is used as abscissa.

These curves were computed from the formulas

$$
\begin{gathered}
\left|\frac{\tilde{\xi}_{O L}}{\xi_{0}}\right|=\left[\frac{2}{\cosh 2 c_{1}+\cos _{\omega} 2 c_{2}+2 c_{3} \sinh 2 c_{1}-2 c_{4} \sin 2 c_{2}+\left(c_{3}^{2}+c_{4}^{2}\right)\left(\cosh 2 c_{1}-\cos 2 c_{2}\right)}\right]^{1 / 2} \\
\tan \delta_{O}=\frac{\tanh c_{1} \tan c_{2}+c_{3} \tan c_{2}+c_{4} \tanh c_{1}}{1+c_{3} \tanh c_{1}-c_{4} \tan c_{2}} \\
\end{gathered}
$$

where

$$
\begin{aligned}
c_{1} & =\left[1-\sin c_{5}\right]^{1 / 2}\left[\frac{\chi_{T 0}}{2}\left|F_{1}\right|\right]^{1 / 2} \\
c_{2} & =\left[1+\sin c_{5}\right]^{1 / 2}\left[\frac{\chi_{T 0}}{2}\left|F_{1}\right|\right]^{1 / 2} \\
c_{3} & =\left(\cos c_{6}\left[1-\sin c_{5}\right]^{1 / 2}-\sin c_{6}\left[1+\sin c_{5}\right]^{1 / 2}\right)\left[\frac{\chi_{T 0}}{2}\left|F_{1}\right|\right]^{1 / 2} \frac{\chi_{T 0}}{\chi_{T 0}}\left|F_{2}\right| \\
c_{4} & =\left(\cos c_{6}\left[1+\sin c_{5}\right]^{1 / 2}-\sin c_{6}\left[1-\sin c_{5}\right]^{1 / 2}\right)\left[\frac{\chi_{T 0}}{2}\left|F_{1}\right|\right]^{1 / 2} \frac{\chi_{I 0}}{\chi_{T 0}}\left|F_{2}\right| \\
F_{1} & =f_{1}+g_{1} j \\
F_{2} & =f_{2}+g_{2} j \\
\cos c_{5} & =\frac{f_{1}}{\left|F_{1}\right|}
\end{aligned}
$$




$$
\begin{aligned}
& \sin c_{5}=\frac{g_{1}}{\left|F_{1}\right|} \\
& \cos c_{6}=\frac{f_{2}}{\left|F_{2}\right|} \\
& \sin c_{6}=\frac{g_{2}}{\left|F_{2}\right|}
\end{aligned}
$$

Equation 116 is valid at all frequencies, and is presented without further explanation for completeness and the use of those with great computational fortitude.

The amplitude ratio and leading phase angle (angle of lead on the time scale of the fundamental where both the fundamental and double frequency waves are cosine terms) of the double frequency distortion at low frequencies (neglecting the end effect) are presented in figures 8 and 9 . The formula used in their computation was

$$
\frac{1}{\xi_{0}}\left(\frac{\tilde{\xi}_{I L}}{\xi_{0}}\right)=\frac{1}{2}\left(\frac{\tilde{\xi}_{O L}}{\xi_{0}}\right)^{2}\left(\frac{c_{1}+j c_{2}}{c_{3}+j c_{4}}\right)
$$

where

$c_{1}=\cosh \left[2 \chi_{T 0}\right]^{1 / 2} \cos \left[2 \chi_{T 0}\right]^{1 / 2}-\cosh \left[\chi_{T 0}\right]^{1 / 2} \cos \left[\chi_{T 0}\right]^{1 / 2}+\frac{\chi_{T 0}}{\chi_{T 0}}\left[\chi_{T 0}\right]^{1 / 2}\left([2]^{1 / 2} \sinh \left[2 \chi_{T 0}\right]^{1 / 2} \cos \left[2 \chi_{T 0}\right]^{1 / 2}-\right.$

$\left.[2]^{1 / 2} \cosh \left[2 \chi_{T 0}\right]^{1 / 2} \sin \left[2 \chi_{T 0}\right]^{1 / 2}-\sinh \left[\chi_{T 0}\right]^{1 / 2} \cos \left[\chi_{T 0}\right]^{1 / 2}+\cosh \left[\chi_{T 0}\right]^{1 / 2} \sin \left[\chi_{T 0}\right]^{1 / 2}\right)+$

$\left(\frac{\chi_{T 0}}{\chi_{T 0}}\right)^{2} \chi_{T 0}\left(-\sinh \left[2 \chi_{T 0}\right]^{1 / 2} \sin \left[2 \chi_{T 0}\right]^{1 / 2}+\sinh \left[\chi_{T 0}\right]^{1 / 2} \sin \left[\chi_{T 0}\right]^{1 / 2}\right)$

$c_{2}=\sinh \left[2 \chi_{T 0}\right]^{1 / 2} \sin \left[2 \chi_{T 0}\right]^{1 / 2}-\sinh \left[\chi_{T 0}\right]^{1 / 2} \sin \left[\chi_{T 0}\right]^{1 / 2}+\frac{\chi_{T 0}}{\chi_{T 0}}\left[\chi_{T 0}\right]^{1 / 2}\left([2]^{1 / 2} \sinh \left[2 \chi_{T 0}\right]^{1 / 2} \cos \left[2 \chi_{T 0}\right]^{1 / 2}+\right.$

$\left.[2]^{1 / 2} \cosh \left[2 \chi_{T 0}\right]^{1 / 2} \sin \left[2 \chi_{T 0}\right]^{1 / 2}-\sinh \left[\chi_{I 0}\right]^{1 / 2} \cos \left[\chi_{I 0}\right]^{1 / 2}-\cosh \left[\chi_{I 0}\right]^{1 / 2} \sin \left[\chi_{I 0}\right]^{1 / 2}\right)+$

$\left(\frac{\chi_{I 0}}{\chi_{T 0}}\right)^{2} \chi_{T 0}\left(\cosh \left[2 \chi_{T 0}\right]^{1 / 2} \cos \left[2 \chi_{T 0}\right]^{1 / 2}-\cosh \left[\chi_{T 0}\right]^{1 / 2} \cos \left[\chi_{T 0}\right]^{1 / 2}\right)$

$c_{3}=\cosh \left[\chi_{T 0}\right]^{1 / 2} \cos \left[\chi_{T 0}\right]^{1 / 2}+\frac{\chi_{T 0}}{\chi_{T 0}}\left[\chi_{T 0}\right]^{1 / 2}\left(\sinh \left[\chi_{T 0}\right]^{1 / 2} \cos \left[\chi_{T 0}\right]^{1 / 2}-\cosh \left[\chi_{T 0}\right]^{1 / 2} \sin \left[\chi_{T 0}\right]^{1 / 2}\right)$

$c_{4}=\sinh \left[\chi_{T 0}\right]^{1 / 2} \sin \left[\chi_{T 0}\right]^{1 / 2}+\frac{\chi_{T 0}}{\chi_{T 0}}\left[\chi_{T 0}\right]^{1 / 2}\left(\sinh \left[\chi_{T 0}\right]^{1 / 2} \cos \left[\chi_{T 0}\right]^{1 / 2}+\cosh \left[\chi_{T 0}\right]^{1 / 2} \sin \left[\chi_{T 0}\right]^{1 / 2}\right)$

The author expresses his appreciation to D. P. Johnson for his assistance in the mathematical development.

Washington, August 2, 1949. 